\title{
Polymers in a Weak Random Potential in Dimension Four: Rigorous Renormalization Group Analysis
}

\author{
D. Iagolitzer ${ }^{1}$, J. Magnen ${ }^{2}$ \\ 1 Service de Physique Théorique, C.E. Saclay, F-91191 Gif-Sur-Yvette, France \\ 2 Centre de Physique Théorique, CNRS, UPR 14, Ecole Polytechnique, \\ F-91128 Palaiseau Cedex, France
}

Received: 5 August 1992/in revised form: 7 July 1993

\begin{abstract}
Correlation functions of the Edwards model of polymers at weak coupling are defined and studied at the critical point, in dimension four, by a rigorous renormalization group method which validates, at any order, perturbative renormalization group results on their behaviour at large distances. Remainders are controlled by a new argument which enlarges the use of methods of constructive field theory to models of statistical physics.
\end{abstract}

\section{Contents}

1. Introduction . . . . . . . . . . . . . . . . . . . . . . . . . . . . . 85

2. The Model and Results . . . . . . . . . . . . . . . . . . . . . . 88

3. Infinite-volume Limit in the Theory with IR and UV Cut-Off $\quad . \quad$. . . . . . . 91

4. The Infrared limit: Introduction c . c c c . c c c . . . . . 96

5. Phase-Space Expansion: Preliminaries . . . . . . . . . . . . . . . 100

6. Renormalization ..... . . . . . . . . . . . . . 105

7 Resummation of Low Momentum Contributions . . . . . . . . . . . . . 108

8. Bounds, Convergence, Large Distance Behaviour ․ . . . . . . . . . . . . . 111

References . . . . . . . . . . . . . . . . . . . 121

\section{Introduction}

Edwards model [1] of polymers in a random potential and the alternative perturbatively equivalent [2, 4] Edwards model [2] of (possibly weakly) self-avoiding polymers play an important role in polymer theory. From the viewpoint of perturbative field theory, they coincide $[3,4]$ with $\varphi^{4}$ "at $N=0$ components" (i.e. $N$ is fixed at zero in perturbative formulae which a priori apply to strictly positive integer values of $N$ ). At the critical point (i.e. for theories with arbitrary size of the polymers) the basic

A large part of this work has also included the collaboration of D Arnaudon 
question is the behaviour of relevant quantities for large distances, or for large size of the polymers, which corresponds from the viewpoint of field theory to the infrared limit. In this paper, correlation functions of the above Edwards models are defined and studied at the critical point, in dimension four, by a rigorous renormalization group method which shows that perturbative renormalization group results are correct at any order, and provides in fact a rigorous control of remainders for sufficiently small couplings (depending on the order). Dimension four is not physical. However the stable fixed point of lower dimension is non-trivial, the large distance behaviour has an anomalous dimension (see Remark 3 below) and the theory cannot be treated rigorously so far.

Perturbative results of the type we are interested in have been obtained either in the framework of field theory (see [5]) or directly in polymer theory in [6]. (Results of field theory provide desired results at the perturbative level, by simply putting $N=0$ in perturbative formulae for $\varphi^{4}$.) Concerning rigorous results, the situation that arises from previous works is the following. First, results on the Edwards model of (weakly) self-avoiding polymers have been obtained in dimension 2 and 3 in [7] and [8] respectively, but the problem treated so far in these dimensions is the ultraviolet (UV) limit, with a fixed infrared (IR) cut-off corresponding to a maximal finite length of polymers. In works in dimension 4 and above, there is on the contrary so far a fixed UV cut-off (corresponding e.g. to replace continuous space by a given lattice) and the problem treated is indeed the IR limit. For dimension 5 and above the model is superrenormalizable at large distances and the existence and behaviour of the correlation functions are known via the so-called lace expansion [20, 21]. On the other hand in dimension 4 , in which the model is just renormalizable, methods of $[9,10]$, based on probabilistic methods or on correlation inequalities respectively, provide bounds on probabilities of intersections of Brownian paths [9] and in [10] estimates on the $\beta$ function of the Edwards model are established. However, these results do not prove the existence and behaviour of the theory for arbitrary large length of the polymers. (Methods of $[9,10,20,21]$ apply on the other hand to values of the coupling that are not necessarily small.)

Rigorous results related to ours follow in constructive field theory, for "infrared $\varphi_{4}^{4}$ " ( $\varphi^{4}$ in dimension 4 with mass zero and fixed UV cut-off), from the methods of $[11,12]$. However, $\varphi^{4}$ "at $N=0$ components" is no longer a field theory and, in contrast to the perturbative situation recalled above, these methods do not provide rigorous results "at $N=0$." Although the general spirit remains close to that of [11, 12], our methods will thus differ in various respects. In particular, the "domination procedure" used in $[11,12]$ to cure crucial divergence occurring in the analysis of bosonic models like $\varphi^{4}$ (and linked to the divergence of the perturbative series) will be here replaced by a new argument which is in some sense the main point of the proof. This new method should also allow the use of techniques inspired from constructive field theory for the rigorous treatment of other models of bosonic type requiring a renormalization group analysis, either in statistical physics or in field theory.

To be more precise on the scope of this paper, let us now give some further indications on the general physical background and on the type of results to be established. The Edwards model of self-avoiding polymers, introduces an interaction factor:

$$
\exp \left[-g^{2} \int_{0}^{T} \int_{0}^{T} \delta\left(\vec{r}(t)-\vec{r}\left(t^{\prime}\right)\right) d t d t^{\prime}+\alpha T\right]
$$


with $g=\infty$; after one or more renormalization group steps $g$ takes finite values depending on the ultraviolet cutoff. The weakly self-avoiding model corresponds to $g$ small enough. The parameter $\alpha$ depends on $g$ at the critical point. The 2-point correlation function $F(x, y)$ is then formally a functional integral with the usual Brownian measure over all paths $\vec{r}$ subject to the condition $\vec{r}(0)=x, \vec{r}(t)=y$, and a further integration over all possible lengths $T$ of the polymers. The problem of interest to us in that framework, is then, the derivation of results on the large distance behaviour of $F$ as $|x-y| \rightarrow \infty$, as also of $2 n$-point correlation functions. The perturbatively equivalent model of polymers in a random potential can be formally defined by its correlation functions. The 2-point function $F$ is e.g. given formally, up to inclusion of a fixed UV cut-off as described later, by the formula

$$
F(x, y)=\int\left\{\frac{1}{p^{2}+i g \sigma}\right\}(x, y)\left[\exp \int\left(i \alpha \sigma(u)+\frac{\alpha^{2}}{2}\right) d u\right] d \mu(\sigma) .
$$

In (1) $g$ is the coupling constant and $\alpha$ is again a function of $g$ to be chosen in order to obtain the critical theory; $\sigma$ is a random (real-valued) function, $d \mu(\sigma)$ is the normalized quadratic measure of covariance $\delta(x-y)$ (i.e. with propagator 1 in $p$-space) and $\left\{\frac{1}{p^{2}+i g \sigma}\right\}(x, y)$ is the kernel of the operator $((-\Delta)+i g \sigma)^{-1}$; in view of the later introduction of the UV cutoff, this operator will also be written $\left(\frac{1}{C}+i g \sigma\right)^{-1}$, where $C$ is the operator whose kernel $C(x, y)$ is the Fourier transform (in $x-y$ ) of the propagator $\widetilde{C}(p)=1 / p^{2}$ and $\sigma$ is here the operator with kernel $\sigma(x) \delta(x-y)$. At $g=0$ one recovers the usual propagator $1 / p^{2}$ corresponding to Brownian motion. The $2 n$-point function is formally defined similarly, $\{\ldots\}(x, y)$ being replaced by $\prod_{i}\{\ldots\}\left(x_{i}, y_{i}\right)$. (The interaction between the $n$ polymers is generated by the contraction between $\sigma$ 's in denominators.)

Results that will follow from our methods e.g. in the critical or "zero-mass" theory include the existence of correlation functions with the known perturbation series of the Edwards models and their behaviour at large distances e.g.:

$$
F(x, y)=\frac{K}{|x-y|^{2}}\left(1+\frac{\lambda_{1}}{\ln |x-y|}+\lambda_{2} \frac{\ln \ln |x-y|}{(\ln |x-y|)^{2}}+R(x, y)\right) .
$$

In (2) the remainder $R(x, y)$ is well defined and is shown to decrease at large distances at least like $1 /(\ln |x-y|)^{2} ; \lambda_{1}, \lambda_{2}$ are "universal" constants, independent of the bare coupling $g$ and of the ultraviolet cut-off. (This is not the case of the constant $K=1 / \zeta_{\text {ren }}$ : see (78)). Similarly, the (amputated, connected) 4-point function $H\left(\tau x_{1}, \ldots, \tau x_{4}\right)$ behaves as $\frac{1}{-\beta_{2} \ln \tau}\left[1+\frac{\beta_{3}}{\beta_{2}^{2}} \frac{\ln \ln \tau}{\ln \tau}+\cdots\right]$, where $\beta_{2}, \beta_{3}$ are standard constants of the model (also independent of $g$ and of the ultraviolet cutoff).

In this paper, correlation functions will be defined as the limits of functions $F_{\Lambda, \varrho}$ of the form (1) with cutoffs $\Lambda, \varrho$, described in Sect. 2.

Remarks. 1) Our methods allow one to construct also the theory away from the critical point, for any positive mass $m$ ( $\alpha$ depending on $g$ and $m$ ), and to study the approach to the critical point following methods of [19].

2) The leading behaviour in (2) is in cst $/|x-y|^{2}$ rather than $(\ln |x-y|) /|x-y|^{2}$ as would be expected in some related situations. This is linked [13] to the fact that 
the anomalous dimension in 4- $\varepsilon$ dimensions is here proportional to $\varepsilon^{2}$ or, as we shall see, to the fact that the renormalization of the coupling constant is in $g^{3}$ and the wave function renormalization in $g^{4}$.

3) In dimension 3, the two-point function at large distances is expected to decay like:

$$
1 /|x-y|^{1-\eta}
$$

where $\eta$ is the anomalous dimension (instead of $1 /|x-y|$ in the free theory); $\eta$, which is independent of $g$, is quite well known experimentally as well as by numerical simulations, $\varepsilon$-expansion and high temperature expansion [5].

The precise statement of the result is given in Sect. 2 . As we shall see, $F$ is initially defined in a finite box $\Lambda$ and with a further IR cut-off in the propagator. The $\Lambda \rightarrow \infty$ limit, in the theory with both UV and IR cut-off in the propagator, will be studied in Sect. 3. An introduction to the infrared limit is given in Sect. 4. More details on the various procedures will be needed, in particular renormalization and resummation of low momentum contributions are given in Sect. 5 to 7. The subsequent derivation of the existence of the correlation functions in the infrared limit and of results on large distance behaviour is outlined in Sect. 8 .

\section{The Model and Results}

Theorem 1. a) There exists a function of $g: F(x, y, g)$ the perturbation series of which in $g$ is that of the two-point correlation function of the Edwards model at the critical point with the propagator:

$$
\eta(p) / p^{2}
$$

where $\eta$ is a $C^{\infty}$ function, positive, equal to one at the origin and with sufficient decrease at infinity. Moreover the asymptotic behaviour of $F$ is given by Eq. (2).

b) $F$ is $C^{\infty}$ in $g \geq 0$ and is Borel summable.

$F$ is thus the unique function with the above perturbation series, analytic in $\arg g<\pi / 4$ for small enough complex values of $g$, with $n^{\text {th }}$ derivatives bounded uniformly by $\operatorname{cst}^{n}(n !)$.

Theorem 1 is stated here for two-point functions but it extends similarly to $2 n$-point functions.

Part $b$ of the theorem will not be proved in this paper. It follows from the construction given below and from a combinatorial argument in [11]. On the other hand, the proof of part a will be given for a specific cutoff $\eta$, of the form:

$$
\eta(p)=\eta^{\prime}(p) /\left(1+\varepsilon \eta^{\prime}(p)\right), \quad \varepsilon \text { small enough, } \quad \eta^{\prime}=p^{10}\left[\left(1 / p^{10}\right)-1 /\left(p^{10}+1\right)\right],
$$

but the proof can be extended to more general choices.

In order to give meaning from the outset to the expression obtained, one first considers the theory in a finite volume $\Lambda$ in $x$-space: the integrals $\int_{\Lambda} \alpha \sigma(x) d x$ and $\int_{\Lambda} \frac{\alpha^{2}}{2} d x\left(=\frac{\alpha^{2}}{2}|\Lambda|\right)$ are then finite. $\Lambda$ will later tend to infinity. On the other hand, it is convenient to introduce also an auxiliary infrared cut-off, e.g. by using the propagator $\widetilde{C}_{(\varrho)}(p)=p^{8}\left[\left(p^{10}+M^{-10 \varrho}\right)^{-1}-\left(p^{10}+1\right)^{-1}\right]$, where $M>1$ is a given (arbitrary) constant: momenta $p$ are then roughly limited to values such that $|p| \geq M^{-\varrho} ; \varrho$ will later tend to infinity. It is then useful to decompose $C$ in momentum 
space, e.g. in the form:

$$
\begin{aligned}
& \widetilde{C}_{(\varrho)}(p)=\sum_{i=1}^{\varrho} \widetilde{C}^{(i)}(p), \\
& \widetilde{C}^{(i)}(p)=p^{8}\left[\left(p^{10}+M^{-10 i}\right)^{-1}-\left(p^{10}+M^{-10(i-1)}\right)^{-1}\right] .
\end{aligned}
$$

The two-point function for the theory for $\Lambda, \varrho$ is then given at that stage by the formula:

$$
\int\left\{C_{(\Lambda, \varrho)} \frac{1}{1+i g \sigma C_{(\Lambda, \varrho)}}\right\}(x, y) \exp \left\{i \alpha \int_{\Lambda} \sigma(z) d z+\frac{\alpha^{2}}{2}|\Lambda|\right\} d \mu(\sigma),
$$

where $\sigma C_{(\lambda, \varrho)}$ is the operator with kernel $\sigma(x) C_{(\Lambda, \varrho)}(x, y)$, and

$$
C_{(\Lambda, \varrho)}(x, y)=\sum_{i=1}^{\varrho} \int_{\Lambda}\left(C_{(\varrho)}^{(i)}\right)^{1 / 2}(x, z)\left(C_{(\varrho)}^{(i)}\right)^{1 / 2}(z, y) d z \quad \text { if } x, y \in \Lambda
$$

and zero otherwise.

For various technical reasons, our starting point will as a matter of fact be slightly different. Equation (3) is first rewritten in the form (leaving $\Lambda$ and $\varrho$ implicit)

$$
\int\left(B \frac{1}{1+i g A \sigma B} A\right)(x, y) \exp \left\{i \int \alpha \sigma+\int \frac{\alpha^{2}}{2}\right\} d \mu(\sigma)
$$

where the "field" operators A and B have the respective kernels:

$$
\begin{array}{ll}
A_{\Lambda, \varrho}(i, x ; y)=A^{(i)}(x, y) & \text { if } x, y \in \Lambda \text { and zero otherwise, } \\
B_{\Lambda, \varrho}(x ; i, y)=B^{(\imath)}(x, y) & \text { if } x, y \in \Lambda \text { and zero otherwise, }
\end{array}
$$

with $A^{(i)}(x, y)=B^{(i)}(x, y)=\left(C_{(\varrho)}^{(i)}\right)^{1 / 2}(x, y)$, and $A \sigma B$ in (4) is the operator with kernel $\int A(. ; z) \sigma(z) B(z ;) d$.$z , i.e.:$

$$
A \sigma B(i, x ; j, y)=\int A(i, x ; z) \sigma(z) B(z ; j, y) d z .
$$

( $A$ and $B$ are distinguished to make clear later composition rules of operators.)

On the other hand, in view of the treatment of the $\varrho \rightarrow \infty$ limit, it will be technically useful to replace the denominator $1+i g A \sigma B$ by

$$
((1-\varepsilon)+\varepsilon \overrightarrow{\nabla A} \cdot \overrightarrow{\nabla B}+i g A \sigma B
$$

for some fixed small $\varepsilon>0$.

This new denominator would be formally identical to the previous one in the absence of UV cut-off (i.e. with the propagator $1 / p^{2}$ ). This is no longer strictly true in the presence of the UV cut-off, which will not be removed, but it corresponds to a new, slightly different choice of the latter. (In the $\Lambda, \varrho \rightarrow \infty$ limits the UV cutoff will then be the cutoff $\eta$ indicated previously.) For simplicity, we shall also replace $1-\varepsilon$ by 1 in this new denominator. Then the "true" coupling constant is $g /(1+\varepsilon)$.

Our starting point will thus be for the two-point function:

$$
F_{\Lambda, \varrho}(x, y)=\sum_{i, j} F_{\Lambda, \varrho, i, j}(x ; y)
$$


where

$$
\begin{aligned}
& F_{\Lambda, \varrho, i, j}(x ; y)=\int F_{i, j, \Lambda, \varrho}(x, y ; \sigma) \exp \left\{i \int_{\Lambda} \alpha \sigma(z) d z+\int_{\Lambda} \frac{\alpha^{2}}{2} d z\right\} d \mu(\sigma), \\
& F_{\Lambda, \varrho, i, j}(x, y ; \sigma) \\
& =\left(B_{\Lambda, \varrho}(x ; i, .) \frac{1}{1+\varepsilon(\overrightarrow{\nabla A})_{(\Lambda, \varrho)} \cdot(\overrightarrow{\nabla B})_{(\Lambda, \varrho)}+i g A_{(\Lambda, \varrho)} \sigma B_{(\Lambda, \varrho)}} A_{\Lambda, \varrho}(j, . ; y)\right) .
\end{aligned}
$$

In the same way the $2 n$-point function is given by:

$$
\begin{aligned}
& F_{\Lambda, \varrho}\left(x_{1}, \ldots, x_{2 n}\right)=\sum_{i_{1}, i_{2 n}} F_{\Lambda, \varrho, i_{1}, i_{2 n}}\left(x_{1}, \ldots, x_{2 n}\right), \\
& F_{\Lambda, \varrho, i_{1}, i_{2 n}}\left(x_{1}, \ldots, x_{2 n}\right) \\
& \quad=\int \prod_{k=1}^{n} F_{\Lambda, \varrho, i_{k}, j_{2 k}}\left(x_{k} ; x_{2 k} ; \sigma\right) \exp \left\{i \int_{\Lambda} \alpha \sigma(z) d z+\int_{\Lambda} \frac{\alpha^{2}}{2} d z\right\} d \mu(\sigma) .
\end{aligned}
$$

We shall prove:

Theorem 2. For $\varepsilon, g$ sufficiently small, there exists a constant $\alpha(g / 1+\varepsilon)$, corresponding to the theory at the critical point, such that:

$$
\lim F_{\Lambda, \varrho \rightarrow \infty}(x, y)=F(x, y)
$$

exists and the asymptotic behaviour given by (2) holds with:

$$
\mid\left(R(x, y) \mid \leq C(g / 1+\varepsilon)(\ln |x-y|)^{-2} .\right.
$$

Remark. $C(g / 1+\varepsilon) \rightarrow \infty$ when $g \rightarrow 0$.

The proof of Theorem 2 and related results is the content of this paper, and $\alpha$ will be explicitly constructed.

The limits as $\Lambda, \varrho \rightarrow \infty$ of the derivatives in $g$ of $F_{\Lambda, \varrho}$ are formally the successive terms of the perturbative series of the Edwards model. The proof of part a) of Theorem 1 is then achieved by applying our construction to the derivatives of $F_{\Lambda, \varrho}(x, y)$; see [11] for a similar analysis in the case of infrared $\phi_{4}^{4}$.

The following bounds will be useful:

$$
\begin{aligned}
& \left|C^{(\imath)}(x, y)\right| \leq \operatorname{cst} M^{-2 i} \exp \left\{-M^{-i}|x-y|\right\}, \\
& \left|A^{(i)}(x, y)\right| \leq \operatorname{cst} M^{-3 i} \exp \left\{-M^{-i}|x-y|\right\} .
\end{aligned}
$$

First and second derivatives of $A^{(i)}$ will on the other hand satisfy the bounds

$$
\left|\frac{\partial^{\alpha}}{\partial x^{\alpha}} A^{(i)}(x, y)\right| \leq \operatorname{cst} M^{-(3+\alpha) i} e^{-M^{-i}|x-y|},
$$

where $\partial^{\alpha} / \partial x^{\alpha}$ stands for $\partial / \partial x_{v^{\prime}}$ if $\alpha=1$, and $\partial^{2} / \partial x_{v}^{2}$ or $\partial / \partial x_{v} \partial / \partial x_{v}$, if $\alpha=2$.

We also note for later purposes that the $L^{2}$ norm of the function $A^{(i)}\left(x_{0},.\right)$, for any given $x_{0}$, satisfies

$$
\int\left|A^{(i)}\left(x_{0}, y\right)\right|^{2} d y \leq \operatorname{cst} M^{-2 i}
$$




\section{Infinite-Volume Limit in the Theory with IR and UV Cut-Off}

In this section $\varepsilon$ is fixed at zero for simplicity and our aim is to study the $\Lambda \rightarrow \infty$ limit in a theory with fixed UV and IR cutoffs in the propagator. We first state

Proposition 1. For $\varepsilon=0$ and $|g| \leq c(\alpha) M^{-2 \varrho}$, with $c(\alpha)$ small enough $\lim _{\Lambda \rightarrow \infty} F_{\Lambda, \varrho}(x, y)=F_{\varrho}(x, y)$ exists and satisfies the bound:

$$
\left|F_{\varrho}(x, y)\right| \leq \operatorname{cst} \exp \left\{-\operatorname{cst}^{\prime} M^{-\varrho}|x-y|\right\} .
$$

This result is without interest in the $\varrho \rightarrow \infty$ limit (since $|g| \leq c(\alpha) M^{-2} \varrho$ ) and we omit the proof, which is similar to that of Proposition 2 below. As an introduction to the next section, we shall as a matter of fact prove the analogue of Proposition 1 for the two-point function $F_{\Lambda}^{(i)}$ defined by the formula:

$$
\begin{aligned}
F_{\Lambda}^{(i)}(x, y)= & \int\left(B_{\Lambda}^{(i)}(x ; .) \frac{1}{1+i g A_{(\Lambda)}^{(i)} \sigma B_{(\Lambda)}^{(i)}} A_{\Lambda}^{(i)}(. ; y)\right) \\
& \times \exp \left\{\left\{i \alpha_{i} \int_{\Lambda} \sigma(z) d z+\frac{\alpha_{i}^{2}}{2}|\Lambda|\right\} d \mu(\sigma),\right.
\end{aligned}
$$

obtained by putting in (8) $\varepsilon=0$ and $i=j$ and removing all internal summations over momentum indices of the $A$ and $B$ 's (all fixed here to $i$ ); $\alpha_{i}$ will be taken equal to $\alpha_{0} M^{-2 i}$, which is the correct order of this parameter as will be checked in the next sections. We then prove:

Proposition 2. For $|g| \leq \boldsymbol{c}\left(\alpha_{0}, \varepsilon^{\prime}\right)$, with $\boldsymbol{c}\left(\alpha_{0}, \varepsilon^{\prime}\right)$ small enough, uniformly in $i$. $\lim _{\Lambda \rightarrow \infty} F_{\Lambda}^{(2)}(x, y)=F^{(i)}(x, y)$ exists for each $i$ and satisfies the bound:

$$
\left|F^{(i)}(x, y)\right| \leq \operatorname{cst}\left(\varepsilon^{\prime}\right) M^{-2 i} \exp \left\{-\left(1-\varepsilon^{\prime}\right) M^{-i}|x-y|\right\}
$$

with $\operatorname{cst}\left(\varepsilon^{\prime}\right) \rightarrow \infty$ and $c\left(\alpha_{0}, \varepsilon^{\prime}\right) \rightarrow 0$ when $\varepsilon^{\prime} \rightarrow 0$.

Proof. The analysis is based, as in related problems in constructive field theory, on a "cluster expansion" [14]. There will be, however, some specific aspects in our framework. This expansion will be convergent at small coupling, in contrast to the perturbative expansion, and will still provide, as the latter, a set of explicit propagators that will link $x$ and $y$, possibly through intermediate boxes. The cluster expansion that seems simplest for our purposes is an expansion with respect to a paving of $\Lambda$ by boxes $\Delta$, e.g. a (hyper)cubic paving. The size of boxes in this paving is cst $M^{i}$ (i.e. a volume $\mathrm{cst}^{4} \mathrm{M}^{4 i}$ of each box) which is convenient in view of purposes of Sect. 4 to 8 , in connection with the bounds (9), (10). It will ensure bounds of the form

$$
\sum_{\Delta^{\prime} \neq \Delta} \exp \left\{-M^{-i} d\left(\Delta, \Delta^{\prime}\right)\right\}<\mathrm{cst} \text { independent of } i .
$$

In the remainder, the index $i$ will be left implicit unless otherwise stated.

A real variable $h_{\Delta, \Delta^{\prime}}$ is associated to each pair $\left(\Delta, \Delta^{\prime}\right)$ of boxed of $\Lambda\left(\Delta^{\prime} \neq \Delta\right)$. Let $h=\left\{h_{\Delta, \Delta^{\prime}}\right\}$ and let $A(h)=B(h)$ be defined by the relation

$$
\begin{aligned}
& A(h)(x, y)=h_{\Delta, \Delta^{\prime}} A(x, y) \quad \text { if } x \in \Delta, y \in \Delta^{\prime} \\
& \text { or vice-versa with by convention } h_{\Delta, \Delta} \equiv 1 .
\end{aligned}
$$


$F_{\Lambda}(x, y ; h)$ is defined as in (12), with $A, B$ replaced by $A(h), B(h)$ in the denominator $D=1+i g A \sigma B$. The actual $F_{\Lambda}$ is reobtained when all $h_{\Delta, \Delta^{\prime}}$ are equal to one, while fixing some $h_{\Delta, \Delta^{\prime}}$ at zero amounts to decouple $\Delta$ and $\Delta^{\prime}$ : in particular, if $\Lambda$ is divided into two decoupled regions $\Lambda_{1}, \Lambda_{2}\left(h_{\Delta, \Delta^{\prime}}=0\right.$ if $\left.\Delta \in \Lambda_{1}, \Delta^{\prime} \in \Lambda_{2}\right)$, then $1 / D(h)$ factorizes into a corresponding product of orthogonal operators:

$$
\frac{1}{D(h)}=\frac{1}{\left.D(h)\right|_{\Lambda_{1}}} \frac{1}{\left.D(h)\right|_{\Lambda_{2}}} \text {. }
$$

Through Taylor expansion of order 1 around the origin in all variables $h_{\Delta, \Delta^{\prime}}$, we then express $F_{\Lambda}(x, y) \equiv F_{\Lambda}(x, y ;\{1,---, 1\})$ as a sum of terms associated to all sets $P$ of pairs of boxes of $\Lambda$, with integration (from 0 to 1 ) over all $h_{\Delta, \Delta^{\prime}}$ such that $\left(\Delta, \Delta^{\prime}\right) \in P$, all other variables $h_{\Delta, \Delta^{\prime}}\left(\Delta \neq \Delta^{\prime}\right)$ being fixed at zero. For each $P$, the set of pairs of $P$ will link the external points $x$ and $y$. The integrand will be expressed as a sum of contributions in which (due to derivatives in the variables $h_{\Delta, \Delta^{\prime}}$ ) the resolvent $D^{-1}$ is replaced (up to unessential aspects) by operators of the form

$$
D^{-1} g(A \sigma B)_{1} D^{-1} g(A \sigma B)_{2} \ldots \ldots D^{-1} .
$$

The general idea will be to use the fall-off properties of $A$ and $B$ 's in numerators to make all needed summations and obtain bounds on $F_{\Lambda}$ that will be uniform in $\Lambda$ at small enough $g$ (The existence of the $\Lambda \rightarrow \infty$ limit will follow similarly, e.g. via expansions of $F_{\Lambda^{\prime}}-F_{\Lambda}, \Lambda^{\prime} \supset \Lambda$ ). To that purpose, it is convenient to first write the expansion of $F_{\Lambda}$ provided by previous procedures in the form:

$$
\begin{aligned}
& F_{\Lambda}(x, y)=\sum_{P} \sum_{\frac{d}{2} \leq k \leq d}(i g)^{k} \sum_{\begin{array}{c}
\text { sets of triplets }\left(\Delta \Delta^{\prime}, \Delta, \Delta^{\prime \prime}\left(n_{j}\right)\right. \\
\text { compatible with } P ; j=1,{ }^{\prime}, k
\end{array}} \\
& \int_{0}^{1} \cdots \int_{0}^{1} \prod_{\left(\Delta, \Delta^{\prime}\right) \in P} d h_{\Delta, \Delta^{\prime}} \int d z_{1}---\left.d z_{k} \int d \mu(\sigma)\right|_{\Lambda(P)} \sigma\left(z_{1}\right)---\sigma\left(z_{k}\right) \\
& \left.\left\{B\left[\frac{1}{D(h)} V_{1}\left(z_{1}\right) \frac{1}{D(h)} V_{2}\left(z_{2}\right)--V_{k}\left(z_{k}\right) \frac{1}{D(h)}\right] A\right\}\right|_{h_{\Delta, \Delta^{\prime}}=0 \text { if } \Delta, \Delta^{\prime} \notin P, \Delta \neq \Delta^{\prime}} \\
& \prod_{\Delta \in \Lambda(p)} \exp \left\{i \int_{\Delta} \sigma(z) d z+\frac{\alpha^{2}}{2}|\Delta|\right\} \text {. }
\end{aligned}
$$

In (17), $d=|P|$, and $\Lambda(P)$ is the set of boxes that belong to a pair of $P$. The third sum runs over sets of triplets $\left(\Delta^{\prime}, \Delta, \Delta^{\prime \prime}\right)$ of $\Lambda(P)^{3}$, one for each $j=1, \ldots, k$, with for each $j$ specification of one among three classes $\left(n_{j}=1,2\right.$ or 3$)$ corresponding to derivations with respect to $h_{\Delta, \Delta^{\prime}}, h_{\Delta, \Delta^{\prime \prime}}$ or both respectively. In the first class, $\left(\Delta, \Delta^{\prime}\right) \in P$ and either $\left(\Delta, \Delta^{\prime \prime}\right) \notin P$ or $\Delta^{\prime \prime}=\Delta$. In the second, the roles of $\Delta^{\prime}$ and $\Delta^{\prime \prime}$ are exchanged. In the third, $\left(\Delta, \Delta^{\prime}\right)$ and $\left(\Delta, \Delta^{\prime \prime}\right)$ are different pairs of $P$. We also write below $\left(\Delta^{\prime}, \Delta, \Delta^{\prime \prime}\right),\left(\Delta^{\prime}, \Delta, \Delta^{\prime \prime}\right)$ and $\left(\Delta^{\prime}, \Delta, \Delta^{\prime \prime}\right)$ triplets with the further specification of class 1,2 and 3 respectively. A set of triplets is compatible with $P$ if each pair of $P$ occurs once, and only once, as a non-underlined pair (obtained by derivation with respect to the corresponding variable $h_{()}$) in one of these triplets. Finally, the operator $V_{j}\left(z_{j}\right)$ is defined as follows for each $j=1, \ldots, k$. If $n_{j}=3$, it is the operator with kernel $A\left(x, z_{j}\right) B\left(z_{j}, y\right)$ if $x \in \Delta^{\prime}, z_{j} \in \Delta, y \in \Delta^{\prime \prime}$, where $\Delta^{\prime}$, $\Delta, \Delta^{\prime \prime}$, are the boxes of the triplet $\left(\Delta^{\prime}, \Delta, \Delta^{\prime \prime}\right)_{j}$, and zero otherwise. If a pair (.) is 
underlined $\left(n_{j}=1\right.$ or 2 ), there is a further multiplicative factor $h_{()}$(equal to one if the two boxes coincide).

The restrictions of $d \mu(\sigma)$ to $\Lambda(P)$ and of the last product in the integrand to boxes $\Delta$ of $\Lambda(P)$ are due to the following facts:

(i) given $P$, values of $\sigma$ at points $x$ that do not belong to $\Lambda(P)$ occur only in the factor $e^{i \alpha \int \sigma(z) d z}$ (since all $h_{\Delta, \Delta^{\prime}}$ have been fixed at zero in other factors when $\left.\left(\Delta, \Delta^{\prime}\right) \notin P\right)$, and

(ii) the integral

$$
\left.\int \exp \left[i \alpha \int_{\Lambda \backslash \Lambda(P)} \sigma(z) d z+\frac{\alpha^{2}}{2} \int_{\Lambda \backslash \Lambda(P)} d z\right] d \mu(\sigma)\right|_{\Lambda \backslash \Lambda(P)}
$$

where $\Lambda \backslash \Lambda(P)$ is the set of boxes of $\Lambda$ that do not belong to $\Lambda(P)$, is equal to one (as is checked by using, e.g. the equality $\int e^{i \int \sigma(z) f(z) d z} d \mu(\sigma)=e^{-\frac{1}{2}(f, C f)}$, where $C$ is here the covariance of the measure $d \mu(\sigma)$, i.e. $C(x, y)=\delta(x-y))$.

Remaining factors $\exp \left\{i \int_{\Delta} \sigma(z) d z+\frac{\alpha^{2}}{2}|\Delta|\right\}$ in the integrand of (17) will later be bounded in modulus by factors exp $\left[|\Delta| \frac{\alpha^{2}}{2}\right]$ for each box $\Delta$ of $\Lambda(P)$, where $|\Delta|=\operatorname{cst}^{4} M^{4 i}$.

The use of fall-off properties of the $A$ and $B$ 's contained in the operators $V$ 's, and of the bounds $\left\|D^{-1}\right\| \leq 1$, do not yet allow one to get uniform bounds in $\Lambda$ as $|\Lambda| \rightarrow \infty$ :

(i) because we have not made a momentum decomposition of the $\sigma\left(z_{1}\right), \ldots, \sigma\left(z_{k}\right)$ "fields" in the numerator of (16), and thus these $\sigma$ have not momentum centered around $M^{-i}$.

(ii) because there are a priori too many terms, due to the $k$ ! possible permutations of numerators $V_{1}, \ldots, V_{k}$, for each given (non-ordered) set of triplets.

Removal of $\sigma$ 's. The most convenient method to treat the first problem is to remove all factors $\sigma\left(z_{1}\right), \ldots, \sigma\left(z_{k}\right)$ from numerators. This can be achieved through contractions of these $\sigma$ 's either together or with those of the denominators $D$ or with $\exp i \alpha \int \sigma$, namely through repeated use of the usual formula $\int \sigma(z) F(\sigma) d \mu(\sigma)=$ $\int\left(\frac{\delta}{\delta \sigma(z)} F(\sigma)\right) d \mu(\sigma)$ (where the fact that $d \mu(\sigma)$ has covariance $\delta(x-y)$ has been used).

The result is analogous to (17), with now a number $k+1$ of resolvents $D(h)^{-1}$ (separated by $k$ operators $V().\left(z_{j}\right)$ ) which can be larger than $d+1$ (namely $d / 2 \leq k \leq$ $2 d$ ) as the consequence of possible contractions of $\sigma(z)$ with $\sigma$ 's involved in $D^{-1}$. These contractions yield new operators $V_{j}\left(z_{j}\right)$ associated with triplets $\left(\Delta^{\prime}, \Delta, \Delta^{\prime \prime}\right)$ (without constraint on the boxes $\Delta^{\prime}, \Delta, \Delta^{\prime \prime}$ in $\Lambda(P)$ ). These triplets belong by definition to class $4\left(n_{j}=4\right)$. On the other hand, $\delta$-functions $\delta\left(z_{\alpha}-z_{\beta}\right)$ "pairing together" two operators $V$ 's are obtained from contraction of $\sigma$ 's (of numerators) either together or with those of $D^{-1}$. In particular any triplet in class 4 is thus associated with a given triplet $\left(\widehat{\Delta}^{\prime}, \Delta, \widehat{\Delta}^{\prime \prime}\right)$ in class 1,2 and 3 . Points $z_{\alpha}$ which are not paired with other points $z_{\beta}$ can arise from contractions with $\exp i \alpha \int \sigma$, in which case multiplicative factors $i \alpha$ are obtained. 
Cauchy Formula. The second problem can be treated by regrouping together terms $D^{-1} V_{(.)} D^{-1} V_{()} \ldots$ associated with all permutations corresponding to a given, nonordered, set of triplets. To that purpose, their sum will be written in the form

$$
\left[\left(\prod_{j=1}^{k} \frac{\partial}{\partial \gamma_{j}}\right) \frac{1}{D(h)+\sum_{j=1}^{k} \gamma_{j} V_{j}\left(z_{j}\right)}\right]_{\gamma_{j}=0, j=1, \quad, k},
$$

and will in turn be expressed (via a Cauchy formula) as an integral over a suitable set of contours, chosen to be circles of radius $r_{j}$ around the origin in the space of complex $\gamma_{j}$ 's. As will appear below, a convenient choice of the $r_{j}$ 's is:

$$
r_{\jmath}=\chi n\left(\Delta_{\jmath}\right)^{-1} M^{2 i} \exp \left\{M^{-i}(1-\eta)\left[d\left(\left(\Delta, \Delta^{\prime}\right)_{j}\right)+d\left(\left(\Delta, \Delta^{\prime \prime}\right)_{j}\right)\right]\right\},
$$

with some sufficiently small constant $\chi>0$ and some small given $\eta, 0<\eta<1$ (e.g. $\eta=1 / 10$ ). In (18) $n(\Delta)$ is the number of pairs of $P$ containing $\Delta$ so that, for any box the total number of indices $j$ with $\Delta_{j}=\Delta$ is bounded by $2 n(\Delta)$.

This choice of the $r_{j}$ 's will first ensure that:

$$
\left\|\sum \gamma_{j} V_{j}\right\| \leq 1 / 2,
$$

because uniformly in the $h$ 's (which are all between 0 and 1):

$$
\left|V_{j}(x, y)\right| \leq \operatorname{cst} M^{-6 \imath} \exp \left\{-\left[M^{-\imath} d\left(\Delta_{0}, \Delta_{j}\right)+d\left(\Delta_{\jmath}, \Delta^{\prime \prime}\right)\right] .\right.
$$

Thus $\left\|\left(D+\sum \gamma_{j} V_{j}\right)_{-1}\right\|$ will still be uniformly bounded. On the other hand, (exponential) fall-off factors between boxes will be reobtained from bounds on the integrals over $\gamma_{j},\left|\gamma_{j}\right|=r_{j}$, which will include a product of factors $1 / r_{j}$, in view of the exponentially increasing factors included in the $r_{j}$ 's (as $d\left(\left(\Delta, \Delta^{\prime}\right)_{j}\right)$ and $d\left(\left(\Delta, \Delta^{\prime \prime}\right)_{j}\right)$ tend to infinity). These factors will allow one to make all remaining summations, first over possible positions of boxes $\Delta^{\prime}, \Delta^{\prime \prime}$ belonging to triplets of class 4 and then over the number and position of boxes corresponding to triplets of class 1,2 or 3 , and over the set of all possible $P$ 's. The fact that non-underlined pairs are distinct two by two and form a connected set (linking the external points $x$ and $y$ in $F_{\Lambda}(x, y)$ ) will be used. The uniform bound (13) will then be obtained on $F_{\Lambda}$.

As already mentioned, the actual existence of the $\Lambda \rightarrow \infty$ limit is proved in a similar way, e.g. by treating differences $F_{\Lambda^{\prime}}-F_{\Lambda}, \Lambda^{\prime} \supset \Lambda$.

We give below more details on the proofs of (19) and of the uniform bound (13) on $F_{\Lambda}^{(i)}$.

Proof of (19) Let $H=\sum_{j} \gamma_{j} V_{j}$. The most elementary bound $\|H\|^{2} \leq$ $\int|H(x, y)|^{2} d x d y$ is not adequate. (The right-hand side tends to infinity with $\Lambda$.) We shall instead use the following slightly more refined result which applies to any operator $H$ with (regular) kernel $H(x, y)$.

Lemma $1\|H\| \leq C(H)^{1 / 2}$, where

$$
\begin{aligned}
C(H) & =\operatorname{Sup}_{y} \int d x Q(x)|H(x, y)| \\
Q(x) & =\int\left|H\left(x, y^{\prime}\right)\right| d y^{\prime}
\end{aligned}
$$


Proof. The inequality $|(f, H g)|^{2} \leq C(H)\|f\|^{2}\|g\|^{2}$ is easily derived from the relations

$$
\begin{aligned}
\left|\int \bar{f}(x) H(x, y) g(y) d x d y\right|^{2} & \leq \int|f(x)|^{2} d x \int d x^{\prime}\left|\int H\left(x^{\prime}, y\right) g(y) d y\right|^{2} \\
\text { and }\left|g\left(y^{\prime}\right) g\left(y^{\prime \prime}\right)\right| & \leq \frac{1}{2}\left(\left|g\left(y^{\prime}\right)\right|^{2}+\left|g\left(y^{\prime \prime}\right)\right|^{2}\right)
\end{aligned}
$$

The bound (19) follows easily: it is shown that $C(H)$ is finite when $H=\sum \gamma_{j} V_{j}$ and $\left|\gamma_{j}\right|=r_{j} ; C(H)^{1 / 2}$ is moreover smaller, e.g. than $1 / 2$ if the constant $\chi$ included in the definition of $r_{j}$ is small enough. We explain below how bounds on $Q(x)$, uniform in $x$, can be obtained. The bound on $C(H)$ follows similarly. The result is based on the exponential fall-off factors $e^{-M^{-i} d\left(\left(\Delta, \Delta^{\prime \prime}\right)_{j}\right)}$ in the bounds on the $A$ and $B$ 's involved in each $V_{j}$. Similar exponential fall-off factors still hold for $r_{j} V_{j}$, in spite of the exponential increase of $r_{j}$, and will allow one to obtain uniform bounds on the sum, independent of the number of terms.

More precisely:

$$
\begin{aligned}
\int d y \sum_{j} r_{j}\left|V_{j}(x, y)\right| \leq & \operatorname{cst} \sum_{\Delta} n(\Delta)^{-1} \sum_{\jmath, \Delta_{j}=\Delta} \int_{y \in \Delta^{\prime \prime}} d y M^{-6 i} M^{2 i} \\
& \times \exp \left\{-\eta\left[M^{-i} d\left(\Delta_{0}, \Delta_{j}\right)+d\left(\Delta_{j}, \Delta^{\prime \prime}\right)\right]\right\}
\end{aligned}
$$

where $\Delta_{0}$ is the box that contains $x$. Since the total number of $j$ with $\Delta_{j}=\Delta$ is bounded by $2 n(\Delta)$,

$$
\int d y \sum_{j}\left|r_{j} V_{j}(x, y)\right| \leq \operatorname{cst} \sum_{\Delta} \exp \left\{-\eta M^{-i} d\left(\Delta_{0}, \Delta\right)\right\} .
$$

By scaling $\sum_{\Delta} \exp \left\{-\eta M^{-i} d\left(\Delta_{0}, \Delta\right)\right\}$ is bounded by a constant independent of $i$ (and $\Delta_{0}$ ). Q.E.D.

Proof of (13). We finally give some indications on the subsequent proof of (13). It is first convenient to write $F_{\Lambda}(x, y)$ as a sum of contributions $F_{(P, k)}$, where $k$ is the number of operators $V$ obtained after removal of $\sigma$ 's from numerators. $F_{(P, k)}$ is written, following previous formulae, as the integral

$$
F_{P, k}(x, y)=\int F_{P, k}\left(z_{1}, \ldots, z_{k}, x, y\right)\left[\delta\left(z_{\alpha}-z_{\beta}\right)\right] d z_{1}, \ldots d z_{k},
$$

where the $\delta$ functions are those pairing points $z_{\alpha}, z_{\beta}$. It is then shown that

$$
\begin{aligned}
& \left|F_{P, k}\left(z_{1}, \ldots, z_{k}, x, y\right)\right| \\
& \quad<M^{-2 i}\left(\operatorname{cst} g M^{-2 i}\right)^{k} \prod_{\Delta \in \Lambda(P)} n(\Delta)^{2 n(\Delta)} \prod_{\left(\Delta, \Delta^{\prime}\right) \in P} \exp \left\{-\operatorname{cst} M^{-i} d\left(\Delta, \Delta^{\prime}\right)\right\},
\end{aligned}
$$

as a direct consequence of bounds of the form

$$
\left\|B^{(i)}(x, .)\right\|\left\|A^{(i)}(., y)\right\|\left\|\left(D+\sum_{\gamma_{\jmath}} V_{j}\right)^{-1}\right\| \prod_{j} 1 / r_{\jmath}
$$

on contributions to $F_{P, k}$. 
A part of the exponential decay is used to compensate the $n(\Delta)^{2 n(\Delta)}$, which is bounded by $\left(n(\Delta) !^{O(1)}\right.$, via the following result:

Lemma 2.

$$
\prod_{\Delta}(n(\Delta) !)^{0(1)} \prod_{\text {pairs }\left(\Delta, \Delta^{\prime}\right)} \exp \left\{-\operatorname{cst} M^{-\imath} d\left(\Delta, \Delta^{\prime}\right)\right\} \leq \prod_{\Delta} O(1) .
$$

Lemma 2 which is proved in Lemma 18.7.2 in [14] (see also Lemma III.1.3 in [15]) allows one to get rid of unwanted factorials $n(\Delta)$ ! via the use of a small part of the available exponential fall-off factors. The (fixed) constant $0(1)$ for each relevant box $\Delta$ (appearing in the right-hand side of (25)) will not cause problems for $g$ sufficiently small.

One gets in turn, for any $\Lambda_{0}$ such that $x$ and $y$ belong to boxes of $\Lambda_{0}$,

$$
\begin{aligned}
& \left|\sum_{P ; \Lambda(P)=\Lambda_{0}} F_{P}(x, y)\right| \\
& \quad<\operatorname{cst} \sqrt{(g)^{\left|\Lambda_{0}\right|-2}} M^{-2 \imath} \exp \left[-c s t^{\prime} M^{-i} L\left(\left\{\Delta, \Delta \in \Lambda_{0}\right\}\right)\right],
\end{aligned}
$$

where $F_{P}=\sum_{k} F_{P, k}$ and $L$ is the minimal length of all trees joining the boxes of $\Lambda_{0}$. Since $L$ is larger than $|x-y|$, a common exponential fall-off $e^{-c s t^{\prime \prime} M^{-i}|x-y|}$ can be factored out. The remaining factors allow one, in the usual ways, to sum over all $\Lambda_{0}$, i.e. all number and positions of boxes [16]. (To that purpose, one may, e.g. replace the factor corresponding to the minimal tree by a sum over all trees joining boxes of $\Lambda_{0}$.)

\section{The Infrared Limit: Introduction}

The method of Sect. 3 can be applied to the theory with propagator $C_{(\varrho)}$ for any given $\varrho$, and this proves Proposition 1. However the latter applies to couplings $g$ less than cst $M^{-2 \varrho}$ and is thus without interest in the $\varrho \rightarrow \infty$ limit. On the other hand, results of Sect. 3 for each $i$, apply to couplings whose maximal values are now uniform in $i$. (As we have seen, this is because, in the bounds on $C^{(2)}(x, y)$, the factor $M^{-2 i}$ is in direct correspondence with the rate $M^{-i}$ of exponential decay in $|x-y|$.) In order to keep the benefit of this result, we are thus led to consider the actual theory as a set of theories, for all values of $i$, that are coupled together. This is achieved via a "multiscale analysis", or phase-space expansion, which will be obtained through the momentumspace decompositions of $A$ and $B$ in terms of $A^{(i)}$ and $B^{(\imath)}$ 's. Cluster expansions will be made, for each $i$, with respect to a paving $\mathbb{D}_{i}$ of $\Lambda$ by boxes of size $M^{i}$, as in Sect. 3, and as becomes now crucial in this section, in agreement with renormalization group ideas. Further (Taylor) expansions with respect to couplings between boxes of different slices will moreover be performed as in related problems for bosonic models of field theory. Besides "horizontal" variables $h^{(\imath)}:\left\{h_{\Delta, \Delta^{\prime}}^{(i)}, \Delta \neq \Delta^{\prime}, \Delta, \Delta^{\prime} \in \mathbb{D}_{\imath}\right\}$ for each $i$ a complementary set of "vertical" variables $v^{(\imath)}$, with one variable $v_{\Delta}^{(i)}$ for each box $\Delta$ of $\mathbb{D}_{i}$, is introduced to that purpose, and we define: $v^{(i)}(x)=v_{\Delta}^{(i)}$ if $x \in \Delta$, $\Delta \in \mathbb{D}_{i}$. Fixing one $v_{\Delta}^{(i)}$ at zero will now amount to decouple locally, in $\Delta$, slices $j \leq i$ from slices $j^{\prime} \geq i+1$.

Finally, in view of getting desired factorization properties between slices, (nonindependent) random variables $\sigma_{i}$ will be introduced for each $i$, with a corresponding 
probability measure $d \mu(\vec{\sigma}, v)$ that will depend on the variables $v, \vec{\sigma}=\left(\sigma_{1}, \sigma_{2}, \ldots\right)$. Although other approaches can be considered, it seems most convenient to keep here the "ultralocal" character of all these $\sigma^{\prime} s$ : the covariance of the $\sigma$ 's will be:

$$
\begin{aligned}
\int \sigma_{j}(x) \sigma_{k}(y) d \mu(\vec{\sigma}, v) & =\delta(x-y) \prod_{m=j}^{k-1} v^{(m)}(x) & & \text { for } j<k \\
& =\delta(x-y) & & \text { for } j=k
\end{aligned}
$$

and the vertex at point $z$ with legs of indices $i$ and $j: A(i, x ; z) \sigma(z) B(z ; j, y)=$ $A^{(i)} \sigma B^{(j)}$ is replaced by: $A^{(i)} \sigma_{\inf (i, j)} B^{(j)}$. (The introduction of these new $\sigma$ 's will not modify the original correlation function when all $h$ and $v$ 's are equal to one.)

Details on these general aspects of the phase-space expansion will be given in Sect. 5. At that stage, the result is an expansion of correlation functions as a sum of diagrams which are here sets of boxes (in various slices) with explicit links produced by derivations with respect to horizontal and vertical variables: explicit $A$ and $B$ 's in each slice and links between slices when $A$ or $B$ 's of different slices are attached to the same vertex $z$ (here $A$ and $B$ 's are those of the numerators before the Cauchy formula).

However, in order to obtain a uniformly convergent expansion, two a priori possible sources of divergences, which were not present in Sect. 3 and are linked respectively with renormalization problems and with the divergence of the perturbative series, will have to be treated:

1) For each $i$, we shall have to consider connected parts in slices $\leq i$ with outgoing legs $A$ or $B$ 's in slices $>i$. Problems linked with renormalization occur because the number of outgoing legs may be less than 5 .

2) Even though the number of explicit $A$ and $B$ 's occurring in each box in a given slice is under control (as in Sect. 3), other $A$ and $B$ 's attached to the same vertices may occur in lower momentum slices and their number, in a given box, may then be arbitrarily large, since e.g. a box in slice $\varrho$ contains $M^{4} \varrho$ boxes of slice 1. (A similar problem occurs for bosonic models of field theory. It is removed for fermionic models in connection with the Pauli principle.)

The treatment of both problems will be inductive, from slice 1 to $\varrho$, with at each stage corresponding reorganizations of the expansion. (Note that a Cauchy formula analogous to that of Sect. 3 is not applied at each stage but will be applied only at the end.) On the one hand, local parts (with 2 and 4 outgoing legs) at the origin of the first problem, will be "absorbed" in a redefinition of effective parameters (depending on the slice $i$ ). This procedure will allow one (as in field theory) to eliminate corresponding divergences if, as will be our case, effective coupling remain uniformily small as $i \rightarrow \infty$.

The second problem comes from the fact that previous procedures have led to expand too much relatively to the low momentum fields $A$ and $B$ 's. To solve this difficulty we will first rewrite at each step $i$ each term of the expansion in a way such that each vertex will now involve only high momentum fields (of index smaller or equal to $i$ ) or only low momentum fields (of index larger than $i$ ), whereas vertices involve initially, at each scale, both low and high momentum fields; this can be achieved up to error terms with better properties. Then we use integration by parts to replace each "half-vertex" $A(., z) B(z,$.$) , where A$ and $B$ are low momentum, by a low momentum $\sigma$ (of momentum roughly smaller than $M^{-i}$ ) that we express as a $\sigma$ averaged in the box of $\mathbb{D}_{i}$ containing $z$. These averaged $\sigma$, in contrast to the $\sigma$ of the vertices $A \sigma B$ coming from derivations in the $h$ 's or $v$ 's, will not be integrated or 
removed and they will have at step $i$ the power counting corresponding to a "halfvertex" $A B$ of slice $i$, i.e.: $M^{-2 \imath}$.

Uniform bounds and convergence are then ensured by methods using, as in Sect. 3, exponential fall-off factors associated in each slice to explicit $A$ and $B$ 's, the smallness of the coupling, and on the other hand exponential fall-off factors $M^{-\left(i^{\prime}-i^{\prime \prime}\right)}$ between slices will allow one to make relevant summations over slices (rigorous "power counting").

Some more precise indications on these two problems are given below, more details being given in Sect. 6 and 7 respectively. The global Cauchy formula and subsequent results are given in Sect. 8.

At each stage $i$ of the inductive procedure, $i+1$ slices denoted below $1,2, \ldots i$ and $i+1$ respectively occur. They correspond roughly to momenta in the regions $1 \geq|p| \geq M^{-1}, M^{-1} \geq|p| \geq M^{-2}, \ldots M^{-(i-1)} \geq|p| \geq M^{-i}$ and $M^{-i} \geq|p|>$ $M^{-\varrho}$ respectively, i.e. more precisely to half-propagators $A^{(j)}$ 's, $j=1, \ldots, i$ and $A_{i+1}=\sum_{k=i+1}^{\varrho} A^{(k)}$, and the analogous $B$ 's. At each stage, a new expression of the (same) 2 point function will be obtained. Taylor expansion will now be made around the origin in all variables $h_{\Delta, \Delta^{\prime}}^{(i)}$, and $v_{\Delta}^{(i)}, \Delta \in \mathbb{D}_{i}$. The order of the expansion in each $h_{\Delta, \Delta^{\prime}}^{(i)}$ can again be chosen equal to one. It will be chosen, e.g. equal to 5 in the $v_{\Delta}^{(i)}$ 's in view of making explicit 2 and 4 point functions of slice $i$ (i.e. functions with a total number of 2 or 4 outgoing $A$ and $B$ 's occurring in slice $i+1$ ) to which "renormalization" will apply; this will allow one to compute coefficients $\alpha_{i}$, $\delta g_{i}=g_{i+1}-g_{i}$ (with $g_{1}=g$ ) and $\delta \varepsilon_{i}=\varepsilon_{\imath+1}-\varepsilon_{i}$ (with $\varepsilon_{1}=\varepsilon$ ). The coefficients $\alpha_{i}, \delta g_{i}$ and $\delta \varepsilon_{i}$ correspond to mass, coupling constant and "wave function" renormalization respectively, and will be defined inductively in terms of $g$ in a way such that the 2 and 4 point functions, as also the second derivative of the 2 point function, are equal to zero at zero momentum. These conditions will ultimately define $\alpha=\alpha(g)=\sum_{i=1}^{\infty} \alpha_{i}$.

Concerning the second problem, the "domination" procedure used in $[11,12]$ in the treatment of infrared $\varphi_{4}^{4}$ (and making recourse to the minus sign in the exponent of the factor $\exp \left\{-\lambda \int \varphi^{4}(x) d x\right\}$ involved in the functional integral that defines the model) is no longer relevant. Here, we shall systematically proceed to "resummation of low momentum contributions," namely we shall remove, at each stage $i, A$ and $B$ 's (as also their first derivatives) of the last slice $i+1$. This will be achieved by simple techniques, in particular "integration by parts" as already indicated. One will finally be left with contributions in which the numerators involve only:

(i) "Well localized fields" $A, B$, or their first derivatives, of indices less than or equal to $i$. (Their spatial exponential fall-off factors will allow summations over boxes of $\mathbb{D}_{i}$.)

(ii) Second derivatives of $A$ and $B$ 's of arbitrary index $k=1,2 \ldots, i+1$. They decrease at least like $|x-y|^{-5}$ so that summations over boxes of $\mathbb{D}_{i}$ are still possible, whatever $k$ is. (The actual method used later will be different, but relies on similar ideas.)

(iii) Factors $\frac{1}{|\Delta|} \int_{\Delta} \sigma_{()}(z) d z, \Delta \in \mathbb{D}_{i}$ coming from the removal of low momentum vertices after integration by parts. In contrast to actual values of $\sigma$ 's at given points, the "power counting" factor induced by such a mean value in a box of $\mathbb{D}_{i}$ is $M^{-2 i}$ and is in fact satisfactory (it is independent of the index of $\sigma$, here equal to $i+1$, and is a consequence of the ultralocal character of the measure). 
To achieve this, it seems simplest from a practical view point to first remove all $\sigma$ 's from numerators as in Sect. 3, but not the averaged $\sigma$ 's coming from previous removal of low momentum fields. New fields $A, B, \ldots$ of index $i+1$ will then be added to those already produced previously. All of them (apart possibly from two) are then eliminated: pairs $A, B$ are replaced by mean values of the product $A(., z) B(z,$. in boxes $\Delta$ and in turn by mean values of $\sigma$ 's, modulo contributions involving first derivatives of $A$ or $B$. The treatment of the latter amounts to dominate $\nabla A \nabla B$ vertices by the $\varepsilon \nabla A \nabla B$ vertices introduced in the ansatz (8); this will be done up to terms involving second derivatives of $A$ and $B$ 's which, as indicated above, will not cause problems. (A related procedure, more satisfactory from a conceptual viewpoint, would be to first replace $\sigma$ 's involved in numerators by their mean values plus fluctuations, and then to treat the latter by contractions).

The methods above, applied inductively from $i=1$ to $\varrho$, yield (as mentioned above) the definition of $\alpha_{i}, g_{i+1}, \varepsilon_{i+1}$ at each step. Relevant bounds, such as: $\left|\alpha_{i}\right| \leq \operatorname{cst} g_{i}^{2} M^{-2 i},\left|\delta g_{i}\right| \leq \operatorname{cst} g_{i}^{3}, \delta \varepsilon_{i} \leq \operatorname{cst} g_{i}^{4}$, and thus $\left|g_{i}\right|<\operatorname{cstinf}\left(g, i^{-1 / 2}\right)$ are established at each step and allow one to establish further uniform bounds and convergence properties of the expansion. In each case, the smallness of all couplings $g_{i}, \varepsilon_{i}$ for small enough $g$ and $\varepsilon$ is used. More precisely, the full factors $g_{\imath}, \varepsilon_{i}$ are not available. Part of them will "disappear"in the replacement of pairs $A, B$ of index $i+1$ by mean values of $\sigma$ 's: integration by parts leads in fact to $\frac{1}{g(.)} \frac{1}{|\Delta|} \int_{\Delta} \sigma_{()}$. Each $A$ or $B$ of the pair is on the other hand associated to a vertex $g_{\left(i^{\prime}\right)} A^{\left(i^{\prime}\right)} \sigma_{()} B_{i+1}$, $i^{\prime} \leq i$, so that a factor $g_{()}^{1 / 2}$ does remain available. (Note that there is a similar loss of $\lambda_{()}^{3 / 4}$ in the domination procedure used in the analysis of infrared $\left.\lambda \varphi_{4}^{4}\right)$. Actual factors remaining available are here $g_{()}, g_{()}^{1 / 2}, g_{()} / \sqrt{\varepsilon_{()}}, \sqrt{\varepsilon_{()}}$, which are still sufficiently small for adequate choices of $g$ and $\varepsilon$.

The more detailed analysis of the flow of $g_{i}$ yields the relation

$$
g_{i+1}=g_{i}+\frac{1}{2} \beta(i) \ln (M) g_{i}^{3}+\gamma(i) \ln (M) g_{i}^{5}+O\left(g_{i}^{6}\right)
$$

in which the remainder is controlled (by the same methods), and where $\beta(i), \gamma(i)$ converge exponentially in $i$, as $i \rightarrow \infty$, towards standard constants $\beta_{2}$ and $\gamma=\beta_{3}-\beta_{2}^{2}$. The coefficients $\beta_{2}$ and $\beta_{3}$ are those such that the Euclidean Feynman diagrams

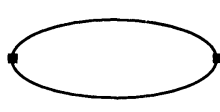

and

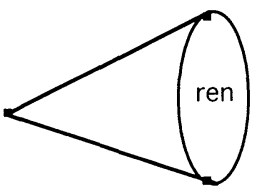

at zero external momenta and cutoff

$M^{-\varrho}$ behave respectively like $\beta_{2} \ln M^{\varrho}$ and $\beta_{3} \ln M^{\varrho}$. As a consequence:

$$
g_{i}=\left[-\beta_{2} i \ln M+\frac{\beta_{3}}{\beta_{2}} \ln i+C^{\prime}\right]^{-1 / 2} \text {. }
$$

Whereas $g_{i}$ then tends to zero as $i \rightarrow \infty$ (asymptotic freedom), all $\varepsilon_{2}^{\prime}$ 's remain close to $\varepsilon$ in view of the bounds $\left|\delta \varepsilon_{i}\right|<\operatorname{cst} g_{i}^{4}$. Because $g_{i}=g, C^{\prime}$ is of the order $1 / g^{2}$. Thus $C^{\prime} \rightarrow \infty$ as $g \rightarrow 0$. The expressions obtained for $g_{i}$ and $\delta \varepsilon_{i}$ allow the derivation of formula (2) and related results.

More details on the various aspects that have been mentioned are given in the next sections. Part of them are close to those encountered in field theory (rigorous 
"power counting," analysis of the flow of coupling constants,...) and will only be briefly treated. For more details on those aspects, the reader is referred, e.g. to [15] (where the part on the domination procedure, which is not relevant for our purposes, can be omitted).

Remark. To be fully complete, the dependence on $\Lambda$ should a priori be kept, the $\Lambda \rightarrow \infty$ limit being ultimately treated. This is at the origin of some new problems, besides those occurring in Sect. 3. They are unessential and can be treated in various ways. For conciseness, this will not be discussed.

\section{Phase-Space Expansion: Preliminaries}

We first describe below the measure $d \mu(\vec{\sigma}, v)$ involved at each stage $i$ of the induction. The phase-space expansion obtained at the first step before renormalization and resummation of low-momentum contributions is then presented. The corresponding procedure to be carried out at stage $i$ (after renormalization and resummation of low momentum contributions in previous steps) is outlined at the end.

The measure $d \mu(\vec{\sigma}, v)$ at step $i$. The measure $d \mu(\vec{\sigma}, v), \vec{\sigma}=\left(\sigma_{1}, \ldots, \sigma_{i+1}\right)$, will be defined at each stage $i$ of the inductive procedure by its covariance

$$
\begin{aligned}
\int \sigma_{j}(x) \sigma_{k}(y) d \mu(\vec{\sigma}, v) & =v_{j, k}(x) \delta(x-y), \\
v_{j, k}(x) & =\prod_{m=(\inf (j, k)}^{\operatorname{Sup}(\jmath, k)-1} v_{m}(x) \text { if } k \neq j, \\
v_{j, j}(x) & =1,
\end{aligned}
$$

where $v_{m}(x)=v_{\Delta}^{(m)}$ if $\Delta$ is the box of $\mathbb{D}_{m}$ that contains $x$ : fixing some $v_{\Delta}^{(m)}$ at zero will then decouple locally higher and lower slices $\left(v_{j, k}(x)=0\right.$ if $\left.x \in \Delta, j \leq m<k\right)$. Variables $v_{\Delta}^{(m)}$ will vary between 0 and 1 , so that $d \mu(\vec{\sigma}, v)$ will always be a well defined probability measure: its convariance is of a positive-type as a simple consequence of the identity

$$
\sum_{j, k=1}^{N} a_{\jmath} v_{j k} a_{k}=\sum_{j=1}^{N}\left(1-v_{j-1}^{2}\right)\left[\sum_{k=j}^{N} v_{\jmath, k} a_{k}\right]^{2}
$$

where $v_{0}=0, v_{j, j}=1$ and $v_{j, k}=\prod_{m=\inf (j, k)}^{\operatorname{Sup}(j, k)-1} v_{m}$.

First step of the induction. The first step $(i=1)$ involves $\sigma_{1}, \sigma_{2}$, with $\int \sigma_{1}(x) \sigma_{2}(y) d \mu\left(\sigma_{1}, \sigma_{2} ; v\right)=v_{1}(x) \delta(x, y)$ and $\int \sigma_{\jmath}(x) \sigma_{j}(y) d \mu\left(\sigma_{1}, \sigma_{2} ; v\right)=$ $\delta(x-y), j=1,2$. We rewrite $F(x, y ; \sigma)$

$$
\begin{aligned}
& \left(\left(B_{1}+B_{2}\right)(x ; .) \frac{1}{1+\varepsilon \overrightarrow{\nabla\left(A_{1}+A_{2}\right)} \cdot \overrightarrow{\nabla\left(B_{1}+B_{2}\right)}+i g\left(A_{1}+A_{2}\right) \sigma\left(B_{1}+B_{1}\right)}\right. \\
& \left.\quad \times\left(A_{1}+A_{2}\right)(. ; y)\right)
\end{aligned}
$$


where $A_{1}$ is equal to $A^{(1)}$ and, $A_{2}=\sum_{m=2}^{\varrho} A(m, . ;$.$) respectively and the analogs for$ $B, \nabla A, \nabla B$. Then we make the following changes:

$$
A(., z) \rightarrow A_{1}(., z)+v_{1}(z) A_{2}(., z),
$$

with similar changes for $B, \nabla A, \nabla B$. In each term $g A_{j} \sigma B_{k}$ in the denominator (with so far $j, k=1,2), \sigma$ is then replaced by $\sigma_{\sup (j, k)}, g$ is replaced by $g_{1}(=g)$, and the new term

$$
g_{2} \int d z\left(1-v_{1}(z)^{2}\right) A_{2}(., z) \sigma_{2}(z) B_{2}(z, .)
$$

which vanishes if $v_{1}=1$, is added. Consequently, $g A \sigma B\left(=g \int d z(A(., z) \sigma(z) B(z,)\right.$. is replaced by

$$
\begin{aligned}
& g_{1} \int d z\left[A_{1}(., z) \sigma_{1}(z) B_{1}(z, .)+A_{2}(., z) \sigma_{2}(z) v_{1}(z) B_{1}(z, .)\right. \\
& \left.\quad+A_{1}(., z) \sigma_{2}(z) v_{1}(z) B_{2}(z, .)+A_{2}(., z) \sigma_{2}(z) v_{1}(z)^{2} B_{2}(z, .)\right] \\
& \quad+g_{2} \int d z\left(1-v_{1}(z)^{2}\right) A_{2}(., z) \sigma_{2}(z) B_{2}(z, .)
\end{aligned}
$$

an expression also written in the form

$$
g_{1}\left[A_{1} \sigma_{1} B_{1}+v_{1} A_{2} \sigma_{2} B_{1}+v_{1} A_{1} \sigma_{2} B_{2}+v_{1}^{2} A_{2} \sigma_{2} B_{2}\right]+g_{2}\left(1-v_{1}^{2}\right) A_{2} \sigma_{2} B_{2} .
$$

If $v_{\Delta}^{(1)}=0$ for some box $\Delta$ of $D_{1}$, the integral over $z$ in $\Delta$ reduces to

$$
g_{1} \int_{\Delta} d z A_{1}(., z) \sigma_{1}(z) B_{1}(z, .)+g_{2} \int_{\Delta} d z A_{2}(., z) \sigma_{2}(z) B_{2}(z, .) .
$$

The term $\varepsilon \nabla A . \nabla B$ is replaced by a similar sum (without $\sigma$ 's), namely

$$
\begin{aligned}
& \varepsilon_{1} \int d z\left(\nabla A_{1}(., z)+v_{1}(z) \nabla A_{2}(., z)\right)\left(\nabla B_{1}(z, .)+v_{1}(z) \nabla B_{2}(z, .)\right) \\
& \quad+\varepsilon_{2} \int d z\left(1-v_{1}(z)^{2}\right) \nabla A_{2}(., z) \nabla B_{2}(z, .) .
\end{aligned}
$$

As already indicated $d \mu(\sigma)$ is replaced by $d \mu\left(\vec{\sigma}, v_{1}\right), \vec{\sigma}=\left(\sigma_{1}, \sigma_{2}\right)$. On the other hand, the term $\exp \left[i \alpha \int \sigma+\frac{\alpha^{2}}{2} \int\right]$ is replaced by

$$
\exp i\left\{\alpha_{1} \int \sigma_{1}(u) d u+\widehat{\alpha}_{2} \int \sigma_{2}(u) d u+\frac{\alpha_{1}^{2}}{2} \int d u+\frac{\widehat{\alpha}_{2}^{2}}{2} \int d u+\alpha_{1} \widehat{\alpha}_{2} \int v_{1}(u) d u\right. \text {. }
$$

As explained below, $\alpha_{1}$ will be determined at the first step, while $\widehat{\alpha}_{2}$ will be determined only at later stages and will be equal to: $\alpha_{2}+\alpha_{3}+\cdots$. We note that the expression obtained coincides, as is easily seen, with the previous one if $v_{1}=1$, whereas there is decoupling between slices 1 and 2 if $v_{1}=0$.

A dependence of $A_{1}, B_{1}, \nabla A_{1}, \nabla B_{1}$ on variables $h_{\Delta, \Delta^{\prime}}^{(1)}$ is then introduced as in Sect. 3, namely:

$$
\left.A_{1}(h)(x, y)=h_{\Delta, \Delta^{\prime}}^{(1)} A_{1}(x, y) \quad \text { and } \quad\left(\nabla A_{1}\right)(h)\right\}(x, y)=h_{\Delta, \Delta^{\prime}}^{(1)}\left(\nabla A_{1}\right)(x, y)
$$


if $x \in \Delta, y \in \Delta^{\prime}$ or vice-versa for $\Delta \neq \Delta^{\prime} ; h_{\Delta, \Delta^{\prime}}^{(1)}=1$ for $\Delta=\Delta^{\prime}$. (The definition of $\left(\nabla A_{1}\right)(h)$ given here seems simplest and most convenient. Note that $\left(\nabla A_{1}\right)(h)$ is then different from $\nabla\left(A_{1}(h)\right)$.) The above procedures define $F\left(x, y ; h^{(1)}, v^{(1)}\right)$ with

$$
F(x, y)=F(x, y ;\{1, \ldots 1\},\{1, \ldots 1\}) .
$$

Taylor expansion (of order 1 or 5 in each $h_{\Delta, \Delta^{\prime}}^{(1)}$ and $v_{\Delta}^{(1)}$ respectively) and elimination of $\sigma$ 's from numerators then lead to a sum of contributions with integrands involving resolvents $\frac{1}{D}$ separated by numerators. Examples of numerators obtained before removal of $\sigma$ 's, by derivation in one variable $v_{\Delta}^{(1)}$ are shown in Fig. 1, where $z \in \Delta$ and the dependence on $\Delta^{\prime}, \Delta^{\prime \prime}$ is left implicit. The term shown in figure 1-a is e.g. equal to $g_{1} A_{1} \sigma_{2} B_{2}$. Each derivative in $v_{1}$ yields at least one "leg" $A$ or $B$ of slice 2 .

slice $1 \quad \mathbf{A}_{1}$

slice 2

Fig. 1.
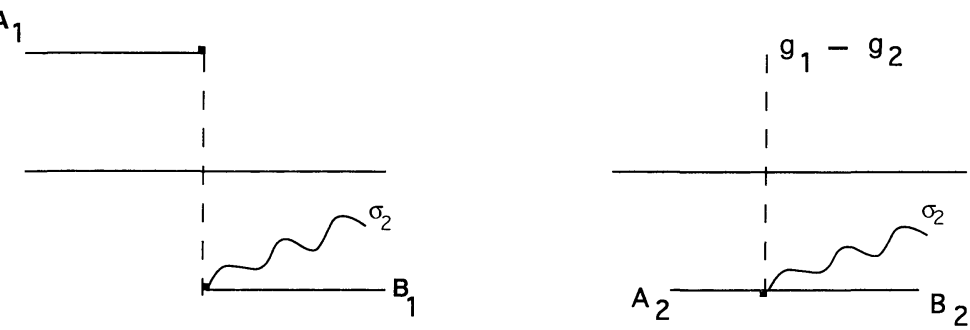

After removal of $\sigma$ 's from numerators, and putting together all $A$ and $B$ 's paired by $\delta$ functions $\delta\left(z^{\prime}-z^{\prime \prime}\right)$, numerators are (after simple regroupings) of the following form:

(i) $\left.\begin{array}{rr}g_{1}^{2} \\ \text { or } & g_{1} g_{2}\end{array}\right) A_{i_{1}}(., z) B_{i_{2}}(z,.) A_{i_{3}}(., z) B_{i_{4}}(z,$. with $\left(i_{1}, \ldots, i_{4}\right)=1$ or 2 and at least one index equal to 1 .

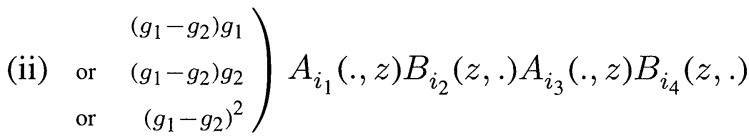

without condition on $\inf \left(i_{1}, \ldots, i_{4}\right)$.

(iii) $\varepsilon_{1} \nabla A_{1} \nabla B_{2}$, or $\varepsilon_{1} \nabla A_{2} \nabla B_{1}$, or $\left(\varepsilon_{1}-\varepsilon_{2}\right) \nabla A_{2} \nabla B_{2}$.

(iv) Other terms arising from the factors $\alpha \int \sigma$ (which are not paired)

or $\left.\begin{array}{r}\left.g_{1}-g_{2}\right)\end{array}\right\}$ (or $\left.\begin{array}{r}\left.A_{2} B_{1}\right)\end{array}\right)\left(\widehat{\alpha}_{2}+v_{1} \alpha_{1}\right)$

$g_{1} A_{1} B_{1}\left(\alpha_{1}+v_{1} \widehat{\alpha}_{2}\right)$

(with e.g. $g_{1} A_{1} B_{2}\left(\widehat{\alpha}_{2}+v_{1} \alpha_{1}\right) \equiv g_{1} A_{1}(., z) B_{2}(z,).\left(\widehat{\alpha}_{2}+v_{1}(z) \alpha_{1}\right)$.

(v) Terms arising from the derivation of the measure $d \mu\left(\vec{\sigma}, v_{1}\right)$ with respect to the variables $v_{\Delta}^{(1)}$ are of the same form as above or of the form: $\alpha_{1} g_{2} A_{2} B_{2}$. 
The vertices (i) to (v) are obtained from the formula

$$
\frac{d}{d v_{\Delta}} \int F(\sigma) d \mu(\sigma, v)=\int_{\Delta} \sum_{j, k} \frac{d v_{j, k}(x)}{d v_{\Delta}}\left(\frac{\delta}{\delta \sigma_{j}(x)} \frac{\delta}{\delta \sigma_{k}(x)} F(\sigma)\right) d \mu(\sigma, v) d x,
$$

where the factor $\delta(x-y)$ of the covariance has been used to put $x=y$ in $\left(\delta / \delta \sigma_{j}(x)\right)$ $\left(\delta / \delta \sigma_{k}(y)\right)$, and where in the first step now considered $\frac{d v_{j, k}(x)}{d v_{\Delta}}$ is equal to one if $x \in \Delta$ and zero otherwise.

The main conclusions are these:

(i) Each numerator is "attached" to slice 1 (i.e. there is at least one $A_{1}, B_{1}, \nabla A_{1}$, $\left.\nabla B_{1}\right)$ apart from terms including factors $\delta g_{1}\left(=g_{2}-g_{1}\right)$ or $\delta \varepsilon_{1}^{\prime}\left(=\varepsilon_{2}-\varepsilon_{1}\right)$ or $\alpha_{1}$ which will be of higher order in $g$, as will appear below.

(ii) Given each box $\Delta$ in $\mathbb{D}_{1}$, there is only, as in Sect. 3, an "essentially finite" number of vertices $z$ involved in numerators: more precisely, this number may be arbitrarily large, as in Sect. 3, since there may be many $A$ or $B$ 's joining $\Delta$ to other boxes, but needed resummations will again be possible due to their exponential fall-off properties.

The following definition and results will be useful in view of the subsequent procedures of Sect. 5.2, 5.3.: for a given contribution, two boxes $\Delta, \Delta^{\prime}$ of $\mathbb{D}_{1}$ such that $h_{\Delta, \Delta^{\prime}}^{(1)}$ is not fixed at zero belong by definition to the same connected component $S$ : boxes in each connected component are linked together by explicit $A$ or $B$ 's (in numerators) of slice 1 . In contrast to Sect. 3, there may be several connected components in slice 1, which are disconnected from each other and are connected together through slice 2: see Fig. 2.

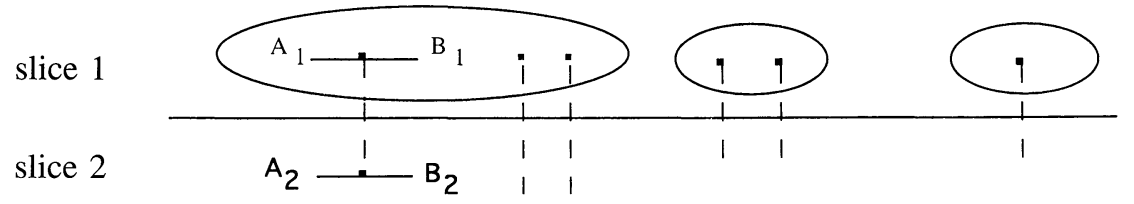

Fig. 2. Several connected components in slice 1 linked by lines --- to slice 2. Legs $A, B$ of slice 1 and 2 , have been left most of the time implicit, as also boxes in slice 1 in each connected component (joined by explicit $A$ or $B$ 's of slice 1).

A connected component $S$ is moreover factorized if $v_{\Delta}^{(1)}$ is fixed at zero for all its boxes $\Delta$. (There may have been previous derivatives in these variables $v$ 's.) Given a factorized connected component, its external legs in slice 2 correspond by definition to $A$ or $B$ 's, or $\nabla A, \nabla B$ 's, of slice 2 belonging to a vertex $z$ attached to slice 1: i.e. there is a corresponding leg of slice 1 in $S$, or the vertex is of the form $\delta g_{1} A_{2} B_{2}$ created by the action of $d / d v_{\Delta}, \Delta \in S$, (after contraction of $\sigma$ 's). The number of external legs is close to the number of links between slice 1 and 2. (It may be somewhat larger in view of the vertices such as $\delta g_{1} A_{2} B_{2}$ with one link and 2 external legs.)

Given sets $S_{1}, S_{2}, \ldots$ of boxes of slice 1 , the following factorization is easily checked if $h_{\Delta, \Delta^{\prime}}^{(1)}=0$ whenever $\Delta, \Delta^{\prime}$ do not belong to the same set, and $v_{\Delta}^{(1)}=0$ for all boxes $\Delta$ of the sets $S_{\beta}, \beta \in \mathscr{L}$ for some $\mathscr{L}$ :

$$
\frac{1}{D}=\left[\prod_{\beta \in \mathscr{C}} \frac{1}{D_{\beta}}\right] \cdot \frac{1}{D^{\prime}}
$$


where $D_{\beta}$ is the restriction of $D$ to $S_{\beta}$ when all $h_{\Delta, \Delta^{\prime}}^{(1)}$ are fixed at zero if $\Delta, \Delta^{\prime}$ do not belong to $S_{\beta}$; and $D_{\beta}^{-1}$ is the inverse of $D_{\beta}$ restricted to $S_{\beta}$.

The expansion obtained so far has still to be modified by the procedures described in Sect. 6, 7 (so far at the first step) before coming to the second step.

General Step. The $i^{\text {th }}$ step is analogous to that above, with by convention:

$$
A_{j}=A^{(j)} \quad \text { for } \quad j \leq i, \text { and } A_{i+1}=\sum_{m=i+1}^{\varrho} A(m, . ; .) .
$$

Each field $A_{i}$ is replaced by $A_{i}+v_{\imath} A_{i+1}$. In the resolvent, $\sigma_{()}$is replaced by $\sigma_{i+1}$ if the highest new index (either of $A$ or $B$ in $A \sigma B$ ) is $i+1$. The new term $g_{i+1}\left(1-v_{i}^{2}\right) A_{i+1} \sigma_{i+1} B_{\imath+1}$ is added. Similarly $\nabla A_{i}$ and $\nabla B_{\imath}$ are replaced by $\nabla A_{i}+v_{i} \nabla A_{i+1}$ and $\nabla B_{i}+v_{i} \nabla B_{\imath+1}$ respectively and a new term $\left(1-v_{i}^{2}\right) \nabla A_{i+1} \nabla B_{i+1}$ is added. The dependence of variables $h^{(i)}$ is then introduced and the various procedures analogous to that above are carried out.

We note that it will be convenient, at step $i$, to adopt the following "weak" definition of a connected component. First a connected component in a strong sense is defined as follows. Two boxes $\Delta, \Delta^{\prime}$ in the same slice $j$ such that $h_{\Delta, \Delta^{\prime}}^{(i)}$ is not fixed at zero belong to the same connected component. Two boxes $\Delta \in \mathbb{D}_{j}$ and $\Delta^{\prime} \in \mathbb{D}_{\jmath+1}$ such that $\Delta \subset \Delta^{\prime}$, and such that there has been at least one derivation $d / d v_{\Delta}^{(\jmath)}$, also belong to the same connected component.

The definition of a connected component in a weak sense is obtained as follows. Given a connected component in a strong sense containing a box of $\mathbb{D}_{k}$, all other boxes $\Delta$ of $\mathbb{D}_{k}$ that contain a box $\Delta^{\prime}$ of $\mathbb{D}_{m}, m<k$, belonging to the connected component in the strong sense are added. The use of connected components in the weak sense allows one, at each stage $i$, to introduce an à la Mayer procedure, see Sect. 6, only relative to the cubes of slice $i$. It will not give problems in the analysis of convergence properties, because exponential fall-off factors in slices with lower indices, will yield required exponential fall-off factors in slices with higher indices, following the usual methods. A factorized connected component is defined as in the first step: all $v_{\Delta}^{(i)}$ are fixed at zero if $\Delta$ belong to a factorized connected component.

We give the $v$ dependence of the resolvent at step $i$ (where the dependence in the $h$ 's is left implicit, $v$ is for $v(x)$ and $v_{0}=0$ by definition):

$$
\begin{aligned}
& \sum_{k=1}^{\imath+1} \sum_{\imath+1 \geq m, j \geq k}\left(1-v_{k-1}^{2}\right) g_{k} v_{k, \inf (m, j)}^{2} v_{\inf (m, j)+1, \sup (m, j)} A_{m} \sigma_{\inf (m, j)} B_{j} \\
& \quad+\sum_{k=1}^{i+1} \sum_{\imath+1 \geq m, j \geq k}\left(1-v_{k-1}^{2}\right) \varepsilon_{k} v_{k, \inf (m, j)}^{2} v_{\inf (m, \jmath)+1, \sup (m, j)} \nabla A_{m} \nabla B_{j} .
\end{aligned}
$$

The term in $\alpha$ is:

$$
\exp \left(\int_{0}^{T} i \sum_{k=1}^{i+1} \alpha_{k} \sigma_{k}(u) d u+\frac{1}{2} \sum_{m, \jmath=1}^{i+1} \alpha_{m} v_{\inf (m, j), \sup (m, j)} \alpha_{j}\right) .
$$

And the expansion consists at each step of a Taylor expansion to the first order in each $h_{\Delta, \Delta^{\prime}}$ and to the fifth order in each $v_{\Delta}$. Then as explained below, an 
à la Mayer procedure is applied to the two and four point functions which are then renormalized, thus defining the values of the effective parameters. The main new ingredient compared to the similar expansion being the replacement, done by integration by parts of the low momentum fields by averages of $\sigma$ fields.

\section{Renormalization}

Renormalization Equations (First Step). Consider a product of the form

$$
\ldots A_{2}(., z) B_{1}(z, .) \frac{1}{D} \ldots \frac{1}{D} A_{1}\left(., z^{\prime}\right) B_{2}\left(z^{\prime}, .\right) \ldots
$$

such that both $z$ and $z^{\prime}$ belong to boxes in a common factorized connected component $S_{\beta}$ and such that all $A$ and $B$ 's (in numerators) between the two resolvents $D^{-1}$ (that have been explicitly exhibited above) belong to slice 1 . In view of (34), it is easily seen that these resolvents can be replaced by $D_{\beta}^{-1}$ and that in fact everything between the points $z, z^{\prime}$ also belongs to $S_{\beta}$. Given a set $\Sigma$ of boxes of slices 1 , let $F_{\Sigma}\left(z, z^{\prime}\right)$ be the sum over all contributions $B_{1}(z,.) \frac{1}{D}-\cdots-\frac{1}{D} A_{1}\left(., z^{\prime}\right)$ of the form above and with the same "support" $\Sigma$, i.e. corresponding to various possible $S_{\beta}$ 's having that support. A first regrouping of terms in the expression of the 2-point function then leads to new contributions whose integrands include now parts of the form

$$
\ldots A_{2}(., z) F_{\Sigma}\left(z, z^{\prime}\right) B_{2}\left(z^{\prime}, .\right) \ldots
$$

with $z, z^{\prime}$ in boxes of the set $\Sigma$. It will be useful to rewrite this part as a sum of terms in which $A_{2}$ and $B_{2}$ are attached to the same point $z$, or $z^{\prime}$, plus contributions involving gradients $\nabla A_{2}, \nabla B_{2}$, taken again at the same point $z$, or $z^{\prime}$, and terms involving at least three derivatives of $A_{2}$ or $B_{2}$,

$$
\begin{aligned}
& A_{2}(., z) F_{\Sigma}\left(z, z^{\prime}\right) B_{2}\left(z^{\prime}, .\right) \\
& \quad=\frac{1}{2} A_{2}(., z) F_{\Sigma}\left(z, z^{\prime}\right) B_{2}(z, .)+\frac{1}{2} A_{2}\left(., z^{\prime}\right) F_{\Sigma}\left(z, z^{\prime}\right) B_{2}\left(z^{\prime}, .\right)+\cdots
\end{aligned}
$$

If we consider e.g. the terms $A_{2}(., z) F_{\Sigma}\left(z, z^{\prime}\right) B_{2}(z,$.$) , we would like to now sum$ over $z^{\prime}$ and all possible supports $\Sigma$ (attached to $z$ ). This is not directly possible because there are non-overlap conditions between boxes belonging to supports of various weakly connected components. This problem can be treated via a procedure à la Mayer, inspired from statistical physics, intended to "free" all boxes in $\Sigma$ (except that containing $z$ ) from other boxes. If two weakly connected supports $\Sigma$ and $\Sigma^{\prime}$ overlap, then it is because they have a box of $\mathbb{D}_{i}$ in common by definition of weak connectedness; so at step $i$ the Mayer procedure concerns only the non-overlapping conditions between boxes of $\mathbb{D}_{i}$. We write each non-overlap factor $\chi\left(\Delta_{\alpha}, \Delta_{\beta}\right)$ between two boxes $\Delta_{\alpha}, \Delta_{\beta} \in \mathbb{D}_{i}\left(\chi=0\right.$ if $\Delta_{\alpha}$ and $\Delta_{\beta}$ coincide, $\chi=1$ if $\left.\Delta_{\alpha} \neq \Delta_{\beta}\right)$ in the form $1+(\chi-1)$. All relevant products are then expanded. A new expansion is then obtained with new links $(\chi-1)$ between some boxes $\left(\chi-1=0\right.$ if $\Delta_{\alpha} \neq \Delta_{\beta}$, $\chi-1=-1$ if $\Delta_{\alpha}=\Delta_{\beta}$ ), besides the links associated to explicit $A$ and $B$ 's (of slice 1 ). Although the new expansion is somewhat more complicated, this does not lead to important problems and this aspect will not be developed here. We only note that some care is needed in the derivation of final bounds: suitable regroupings of terms have to be made. (There would be otherwise too many terms leading to unwanted 
factorials.) See, e.g. the treatment of these problems given in [17]. Possible "Mayer" links between previous connected components will create new contributions with more than two external legs (in slice 2). Here, we are only interested in cases corresponding to two external legs, at the same point $z$ (or $z^{\prime}$ ) of a box $\Delta_{0}$. Resummations previously mentioned then lead to contributions whose integrands will no longer contain parts of the form mentioned at the beginning of this section but instead to parts of the form $A_{2}(., z)\left[\int_{\Delta} d z^{\prime} F^{(1)}\left(z, z^{\prime}\right)\right] B_{2}(z,$.$) , where F^{(1)}\left(z, z^{\prime}\right)$ is here the 2-point function of slice 1 obtained by summation over all possible (connected) components involving $z, z^{\prime}$. The integral $\int d z^{\prime} F^{(1)}\left(z, z^{\prime}\right)$ is convergent in the $\Lambda \rightarrow \infty$ limit, as checked by the methods of Sect. 3. It does not depend on $\Delta_{0}$ or on the point $z$ in $\Delta_{0}$ in that limit, and is equal to $\widetilde{F}^{(1)}(0)$.

The value of $\alpha_{1}$ will be chosen such that $\widetilde{F}^{(1)}(0)=0$ ("mass renormalization"). Contribution terms of lowest order in $g=g_{1}$ (and $\varepsilon$ ) are

(i) $\alpha_{1} g_{2}$, corresponding to the vertex

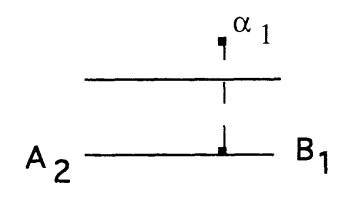

From later analysis, $g_{2}$ is itself equal to $g_{1}$ up to higher order terms in $g$.

(ii) $-c_{1} g_{1}^{2}$, where

$$
c_{1}=\equiv \int B_{1}(z, u) A_{1}(u, z) d u=\int \widetilde{C}^{(1)}(p) d p,
$$

corresponding to a diagram of the form

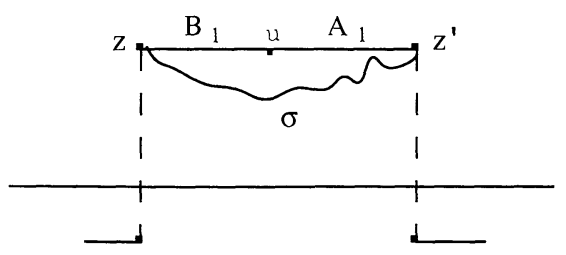

with two vertices that have been identified through contractions of $\sigma$ 's. Namely one starts from an original part of the form

$$
A_{2}(., z)\left[\int d u\left(g_{1} \sigma(z) B_{1}(z, u)\right)\left(g_{1} A_{1}\left(u, z^{\prime}\right) \sigma\left(z^{\prime}\right)\right)\right] B_{2}\left(z^{\prime}, .\right)
$$

where the resolvent $1 / D$ between the two $A \sigma B$ 's has been replaced by its lowest order contribution in $g$, and $\varepsilon$, i.e. 1 . Contractions of $\sigma$ 's yield the $\delta$ function $\delta\left(z-z^{\prime}\right)$. This will provide the first equation:

$$
\alpha_{1} g_{1}-c_{1} g_{1}^{2}+\text { higher order terms }=0 .
$$

Vertices involving gradients are treated similarly. One is then led to the second equation:

$$
\varepsilon_{1}-\varepsilon_{2}-c_{2} g_{1}^{4}+\text { higher order terms }=0,
$$


where $\varepsilon_{1}-\varepsilon_{2}$ is in fact the original factor in front of $\nabla A_{2} . \nabla B_{2}$ and

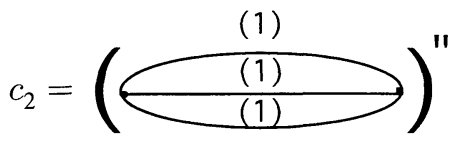

i.e., $c_{2}$ in the second derivative at zero momentum (in momentum space) of the graph with propagators $C^{(1)}$ on each line. This graph is associated after contractions of $\sigma$ 's to the diagram

slice 1

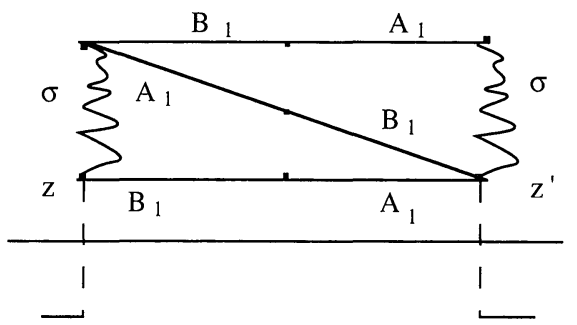

More precisely, the previous mass renormalization has led one to subtract from this diagram its value at zero (in momentum space) with a result of the form $G\left(z, z^{\prime}\right) \nabla A_{2}(., z) \nabla B_{2}(z,$.$) up to terms involving more derivatives. (Note that terms$ with only one gradient vanish.) The coefficient $c_{2}$, obtained after the further procedure now considered, is equal to $\widetilde{G}(0)=\int G\left(z, z^{\prime}\right) d z^{\prime}$.

At this stage, there are no longer 2-point functions except those with a total number of at least 3 gradients (or derivatives) which will not give problems. (One derivative is equivalent to one external leg from the viewpoint of power counting.)

The 4-point function is in turn treated similarly. Points $z$ 's involved are again brought together. A new à la Mayer procedure is also applied. The constant $g_{2}$ is then chosen in a way such that the total 4-point function (attached to a given point $z$ ) is equal to zero.

This leads to the third equation:

$$
g_{1}\left(g_{1}-g_{2}\right)-c^{3} g_{1}^{4}+\text { higher order terms }=0,
$$

where

$$
c_{3}=\overbrace{(1)}^{(1)}(0) \equiv \int d p \widetilde{C}^{(1)}(p)^{2} .
$$

The relevant diagram is in fact here

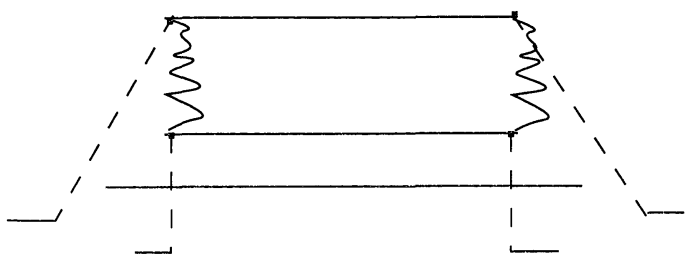

and $c_{3}$ is its value at zero in momentum space (after contraction of $\sigma$ 's). 
Equations (37), (38), (40) are a set of three coupled equations, all of them involving all relevant quantities. However, these equations become decoupled at lowest order, so that simple solutions are obtained. In particular

$$
\begin{aligned}
\alpha_{1} & =c_{1} g_{1}+O\left(g_{1}^{3}\right), \\
\varepsilon_{1}-\varepsilon_{2} & =c_{2} g_{1}^{4}+O\left(g_{1}^{6}\right), \\
g_{1}-g_{2} & =c_{3} g_{1}^{3}+O\left(g_{1}^{5}\right) .
\end{aligned}
$$

Renormalization Equations (General Step). The general procedures are similar to the above. They now yield the following results (with new higher order contributions):

$$
\alpha_{i}=\overbrace{g_{i}-g_{\imath+1}=\underbrace{(i)}_{(i)} g_{i}+O\left(g_{i}^{3}\right),}^{(i)} g_{i}^{3}+O\left(g_{i}^{5}\right),
$$

\section{Resummation of Low Momentum Contributions}

We now describe the procedure of resummation of low momentum contributions at stage $i$, after all previous operations have been carried out. They have allowed one to express, e.g. the 2-point function (for given, arbitrary $\Lambda, \varrho$ ) as a sum of contributions with integrands involving products of operators, namely numerators separated by resolvents $R=1 / D$. Initially, numerators were of the form $A(., z) B(z,$. with the vertex $z$ in a given box $\Delta$ (with possible pairings between vertices) and $\nabla A(., z) \nabla B(z,$.$) , arising from the supplementary term \varepsilon \nabla A \nabla B$ (that has been introduced in $D$ ) through derivations in variables $h$ of $v$ 's. The various procedures that have already been carried out have led to a somewhat different situation (as will appear from the analysis below, concerning resummations of low momentum contributions carried out at earlier stages), but the analysis is not substantially modified. As a matter of fact, numerators may now involve also fields $\partial A, \partial^{2} A$ and $\partial B, \partial^{2} B$ (meaning one or more derivatives of $A$ of $B$ ) and the intermediate point in $A$ and $B$ (or their derivatives) may be distinct although they lie in the same box $\Delta$ (e.g. $A(., z) B((z,$.$) ,$ $z \in \Delta$, may be replaced by $A\left(., z_{\alpha}\right) B\left(z_{\beta},.\right), z_{\alpha}, z_{\beta} \in \Delta$ with possibly $\left.z_{\alpha} \neq z_{\beta}\right)$. This will be made more precise later.

Elimination of Low Momentum $A$ and $B$ 's (in numerators). We start with the elimination of "low momentum" or "badly localized fields" $A_{\imath+1}, B_{i+1}$, and will come back later to the treatment of other ones (derivatives of $A$ and $B$ 's).

Given a term contributing to the 2-point Green function and a box $\Delta$ in slice $i$, let us first consider fields $A_{i+1}(., z), B_{\imath+1}\left(z^{\prime},.\right)$ in numerators with vertices $z, z^{\prime}, \cdots$ 
in $\Delta$. If there is only one $A_{i+1}$, or one $B_{i+1}$, or $A_{i+1}$ and one $B_{i+1}$ involved in $\Delta$, this term is kept without change. Otherwise the following procedures are used:

(i) Reversal procedure. Consider e.g. a term of the form

$$
-\cdots-R A_{i+1}\left(., z_{\alpha}\right) B_{i^{\prime}}\left(z_{\alpha},\right) R---R A_{i+1}\left(., z_{\beta}\right) B_{i^{\prime \prime}}\left(z_{\beta}, .\right)-\cdots--
$$

with $i^{\prime}, i^{\prime \prime} \leq i$. This term can be written in the form

$$
\text { ---RA } A_{i+1}\left(., z_{\alpha}\right) B_{i+1}\left(z_{\beta}, .\right) R---R A_{i}\left(., z_{\alpha}\right) B_{i^{\prime \prime}}\left(z_{\beta^{\prime \prime}}\right) \text {-- }
$$

as a consequence of the relations $R^{\mathrm{tr}}=R$, where tr is transposition, since in particular $(A \sigma B)^{\operatorname{tr}}=A \sigma B$ and $A^{\text {tr }}(., z)=B(., z)$. (These relations, directly checked, are linked to the existence of the square root of $C$ and, as matter of fact, to the stability of the $\varphi^{4}$ theory.)

It will then also be useful, for $z_{\alpha}, z_{\beta}$ integrated in the same box $\Delta$, to write

$$
\begin{aligned}
-- & R A_{i+1}\left(., z_{\alpha}\right) B_{i+1}\left(z_{\beta}, .\right) R-- \\
= & -R\left(\frac{1}{|\Delta|} \int_{\Delta} A_{i+1}(., z) B_{i+1}(z, .) d z\right) R-- \\
& \left.+\ldots-R \int_{0}^{1} d t \frac{1}{|\Delta|} \int_{\Delta}\left(\overrightarrow{z_{\alpha}-z}\right) \vec{\nabla} A^{i+1}\left(., t z_{\alpha}+(1-t) z\right) B_{i+1}\left(z_{\beta}, .\right) d z\right) R-. \\
& +\cdots R \int_{0}^{1} d t \frac{1}{|\Delta|} \int_{\Delta} A_{\imath+1}\left(., z_{\alpha}\right)\left(\overrightarrow{z_{\beta}-z}\right) \\
& \left.\times \vec{\nabla} B_{i+1}\left(t z_{\beta}+(1-t) z, .\right) d z\right) R-. .
\end{aligned}
$$

We recall that gradients (or derivatives) of $A$ 's or $B$ 's will induce a better "power counting."

(ii) Elimination of "low momentum" $A_{i+1}$ and $B_{i+1}$ 's. The following identity, which follows from usual contractions of $\sigma$ 's with those in the denominator of $R$, will be used:

$$
\begin{aligned}
\int & d \mu(\sigma) \sigma_{i+1}(z) R\left(x_{1}, y_{1}\right) \cdots R\left(x_{n}, y_{n}\right) \\
= & -\int d \mu(\sigma) \sum_{m=1}^{n} R\left(x_{1}, y_{1}\right) \cdots R\left(x_{m}, z^{\prime}\right) A_{\imath+1}\left(z^{\prime}, z\right) \widehat{g}_{i+1}(z) B_{i+1}\left(z, z^{\prime \prime}\right) \\
& \times R\left(z^{\prime \prime}, y_{m}\right) \cdots R\left(x_{n}, y_{n}\right) d z d z^{\prime} d z^{\prime \prime} \\
& + \text { terms involving } A \text { and } B \text { 's, one of which at least has index } \leq i
\end{aligned}
$$

where

$$
\widehat{g}_{i+1}(z)=\sum_{m=1}^{i+1}\left(1-\left[v_{m-1}(z)\right]^{2}\right) g_{m}\left[v_{m, i+1}(z)\right]^{2} .
$$

Repeated use of the procedures above allows one to replace all fields $A_{i+1}(., z)$ or $B_{i+1}(z$,$) with vertices z$ in $\Delta$ by products of:

- mean values $\frac{1}{|\Delta|} \int_{\Delta} \sigma_{i+1}\left(z^{\prime}\right) d z^{\prime}$ of $\sigma_{i+1}$ in $\Delta$

- factors $\alpha_{i+1}$ because in the functional integral there is also the $e^{\imath \int \alpha_{\imath+1} \sigma_{\imath+1}}$ term

- factors $M^{-2 i}$ because mean values of $\sigma$ can contract together. 
We get one of these factors for each pair $A_{i+1} B_{i+1}$ that has been removed, at the cost of some multiplicative factors $\left[\frac{1}{|\Delta|} \int_{\Delta} \widehat{g}_{i+1}\left(z^{\prime}\right) d z^{\prime}\right]^{-1}$ and modulo the introduction of "better" terms involving more derivatives of $A_{i+1}$ or $B_{i+1}$ 's (the number of which will remain limited and under control).

It can be shown that the factor $\left[\frac{1}{|\Delta|} \int_{\Delta} \widehat{g}_{i+1}\left(z^{\prime}\right) d z^{\prime}\right]^{-1}$ is less than $1 / g_{i+1}$ by taking into account the fact (to be checked) that $g_{i+1} \leq g_{j}$ if $j \leq i$ and the identity

$$
\sum_{m=1}^{i}\left(1-v_{m-1}^{2}\right) v_{m}^{2}---v_{\imath}^{2}=1 \text {. }
$$

For each vertex involving initially one $A$ or $B$ of index $j \leq i$ and one $A$ or $B$ of index $i+1$, which has been eliminated, factors arising in later bounds from coupling are as follows:

a) a factor $1 / \sqrt{g_{i+1}}$ arising from the elimination of the $A$ or $B$ of index $i+1$ (since the elimination of the latter and of another $B$ or $A$ of index $i+1$ induces a factor $\left.1 / g_{i+1}\right)$.

b) the initial coupling of that vertex, namely.

$$
\sum_{n=1}^{j}\left(1-v_{n-1}(z)^{2}\right) g_{n} v_{n}^{2}(z)----v_{j-1}(z)^{2} v_{j}(z)-\cdots-v_{i}(z) \text {. }
$$

The latter will be bounded by $g_{m(i, z)}$, where

$$
m(i, z)=\inf \left\{k ; \text { no } v_{m}(z) \text { is fixed at zero if } k \leq m \leq i\right\} .
$$

As a result of a) and b) the factor arising in the bounds will be $g_{m(i, z)} / \sqrt{g_{i+1}}=$ $\left(g_{m(i, z)} / g_{i+1}\right)^{1 / 2} g_{m(i, z)}^{1 / 2}$. The factor $g_{m(i, z)}^{1 / 2}$ will be bounded by $g_{1}^{1 / 2}$ and will thus be arbitrarily small if $g$ is small enough. The factor $g_{m(i, z)} / g_{\imath+1}$ is $\geq 1$. It may become large but this fact will remain under control, as will appear in Sect. 8.

Treatment of Remaining Low Momentum Fields. After the elimination, described previously, of low momentum $A$ and $B$ 's, the situation is the following. Apart possibly from at most one $A_{i+1}$ of $B_{i+1}$ for each box $\Delta$ of $\mathbb{D}_{i}$, numerators are either of the form $A\left(., z_{\alpha}\right) B\left(z_{\beta}\right.$, .) with "well localized" $A$ and $B$ 's (of index $\leq i$ ) and $z_{\alpha}, z_{\beta}$ in the same box $\Delta$ of $\mathbb{D}_{i}\left(z_{\alpha}=z_{\beta}\right.$ or $\left.z_{\alpha} \neq z_{\beta}\right)$, or either $A$ or $B$, or both are replaced by derivatives of $A$ and $B$ 's. In the latter case, fields involved may have the index $i+1$. As above, intermediate points may or not coincide but belong to the same box $\Delta$ of $\mathbb{D}_{\imath}$.

Low momentum derivatives of $A$ and $B$ 's cannot be eliminated as previously through the replacement of pairs by mean values of $\sigma$ 's (since e.g. terms in the resolvent in $\nabla A . \nabla B$ do not involve $\sigma$ 's). However, repeated use of some of the procedures above (reversal procedure, replacement of a point $z_{\beta}$ in a box $\Delta$ by $z_{\alpha}$, up to some derivatives, mean values in boxes $\Delta$ ) allows one to get a new expansion; numerators in the new integrands involve only well localized $A$ and $B$ 's or their derivatives, or have one of the two following forms:

$$
\left[\frac{1}{|\Delta|} \int_{\Delta} \nabla A_{i+1}(., z) d z\right]\left[\frac{1}{\Delta} \int_{\Delta} \nabla B_{i+1}(z, .) d z\right]
$$


(ii) numerators of the form $\partial^{2} A\left(., z_{\alpha}\right) \partial^{2} B\left(z_{\beta},.\right)$ involving seconded derivatives of $A$ or $B$ 's of index $i+1\left(z_{\alpha}, z_{\beta}\right.$ in the same box $\Delta$ of $\left.\mathbb{D}_{i}\right)$.

For reasons already explained, terms (ii) will not give problems. Terms (i) will later be "dominated" by similar terms involved in the resolvent, with coefficient $\varepsilon_{i+1}$, arising from the introduction of the supplementary term $\varepsilon \nabla A \nabla B$ at the outset: see Sect. 8 .

We conclude this section with the following remark: in correction terms, one has also to take into account the fact that a $\sigma$ can be contracted with the factor $e^{i \sum \int \alpha_{\jmath} \sigma_{\jmath}}$ [in which case terms of the type $\alpha_{j} \frac{1}{|\Delta|} \int_{\Delta} v_{i+1, j}(u) d u, \Delta \in \mathbb{D}_{i},(j \leq i+1)$ will occur], or with other $\sigma$ 's already present. In the case of a contraction with $\frac{1}{|\Delta|} \int_{\Delta^{\prime} \in D_{j}} \sigma_{j+1}(u) d u$, terms induced involve factors $M^{-4 i} v_{i, j}$ which can be written in the form: $M^{-2 \imath} M^{-2 \jmath} M^{-2(\imath-j)}$ multiplied by a product of $v_{k}$ 's. The factor $M^{-2 i} M^{-2 j}$ corresponds to the correct power counting.

\section{Bounds, Convergence, Large Distance Behaviour}

The aim of this section is to give details (Sect. 8-1, 8-2) on the way uniform bounds on $F$ are now established in the $\varrho \rightarrow \infty$ limit. Convergence is established similarly (from related bounds on $F_{\varrho^{\prime}}-F_{\varrho}, \varrho^{\prime}>\varrho$ ). Large distance properties are proved (in Sect. 8.3) by first extracting explicit contributions of lowest orders and then applying previous methods to obtain bounds on remainders.

Let us summarize the situation arising from previous sections. The inductive procedure, carried out from slice 1 to $\varrho$, yields a sum of terms each of which is associated to a connected component with horizontal and vertical lines joining boxes in various slices. They include indications of the various derivatives in variables $h$ or $v$ 's that have been made and further indications related to the various procedures that have been carried out. Regroupings of terms associated with permutations of numerators are made in Sect. 3. For each multiscale support, one is led to an integral:

over variables $h_{\Delta, \Delta^{\prime}}^{(i)}$ and $v_{\Delta}^{(i)}$ not fixed at zero with the measure

$$
\prod_{i ;\left(\Delta, \Delta^{\prime}\right)} d h_{\Delta, \Delta^{\prime}}^{(\imath)} \prod_{i, \Delta} \frac{1}{4 !}\left(1-v_{\Delta}^{(i)}\right)^{4} d v_{\Delta}^{(i)}
$$

over $\sigma$ 's with the measure $d \mu(\vec{\sigma}, v), \vec{\sigma}=\left(\sigma_{1}, \ldots, \sigma_{\varrho}\right)$,

over vertices $z$ (integrated in relevant boxes), and

over variables $t$ (between 0 and 1) arising from formulae such as (47).

Besides a derivative of the form $\left.\prod_{\alpha} \frac{d}{d \gamma_{\alpha}} \frac{1}{D+\Sigma \gamma_{\alpha} V_{\alpha}}\right|_{\gamma_{\alpha} \equiv 0}$ to be treated as in Sect. 3 by a Cauchy integral over circles of radius $r_{\alpha}$ in the space of complex $\gamma_{\alpha}$ 's, the integrand will include all other factors such as $\left(z^{\prime}-z^{\prime \prime}\right)_{\nu}$, products of variables $v$, of effective couplings $g_{\imath(\mathscr{T})}, \varepsilon_{\imath(\mathscr{V})}, \delta g_{i(\mathscr{W})}$ for the various vertices $\mathscr{V}$ involved, as also mean values $\left(\frac{1}{|\Delta|} \int_{\Delta} \sigma_{\imath+1}\right)^{n(\Delta)}$ and inverse coupling arising as explained in Sect. 7 from the resummation of low momentum contributions. And for each vertex $\mathscr{V}, i(\mathscr{V})$ is the largest index $i$ such that there has been a derivative at that stage. 
Leaving aside unessential factors, one obtains thus as the result of the expansion $F_{i_{1}, i_{2}}$ in the form of a sum of terms:

$$
F_{i_{1}, \ell_{2}}=\sum \int \ldots B^{\left(i_{1}\right)}(., .) \frac{1}{D+\sum_{\alpha} \gamma_{\alpha} V_{\alpha}} A^{\left(i_{2}\right)}(., .)\left(\prod \frac{1}{|\Delta|} \int_{\Delta} \sigma\right) d \mu(\sigma) \prod_{\alpha} d \gamma_{\alpha}
$$

and therefrom a bound of type:

$$
\begin{aligned}
\left\|F_{i_{1}, i_{2}}\right\|= & \sum\left(\prod_{\alpha} \frac{1}{r_{\alpha}}\right)\left\|B^{\left(i_{1}\right)}\right\|\left\|\frac{1}{D+\sum_{\alpha} \gamma_{\alpha} V_{\alpha}}\right\|\left\|A^{\left(i_{2}\right)}\right\| \\
& \times\left(\int\left(\prod \frac{1}{|\Delta|} \int_{\Delta} \sigma\right)^{2} d \mu(\sigma)\right)^{1 / 2} .
\end{aligned}
$$

We first recall the type of operators $V_{\alpha}$ involved and indicate relevant choices of the $r_{\alpha}$ 's which will ensure first (Sect. (8.1)) that $\left\|\left(D+\Sigma \gamma_{\alpha} V_{\alpha}\right)^{-1}\right\|$ is bounded by a constant (e.g. 2) and will allow one to obtain bounds on $F$, uniform in $\varrho$, through bounds on the factors $1 / r_{\alpha}$ (Sect. 8.2):

\subsection{Choice of the $r_{\alpha}$ 's and Bounds on $\left\|\Sigma \gamma_{\alpha} V_{\alpha}\right\|$.}

As in Sect. 3, the dependence in the $h$ 's of the $V$ 's plays no role, since these variables are bounded by one, and e.g.: $\left|A_{\jmath}(x, y, h)\right| \leq M^{-3 \jmath} e^{-M^{-\jmath}|x-y|}$ uniformly in $h$.

We now list the different kinds of $V$ 's and give the corresponding $r$ 's:

a) Operators $V$ 's of the form $A_{j}(., z) B_{k}(z,$.$) or A_{j}\left(., z_{1}\right) B_{k}\left(z_{2},.\right), j, k \leq i+1, z$ or $z_{1}, z_{2}$ in a box $\Delta$ of $\mathbb{D}_{i}(j$ and $k$ equal to $i+1$ for at most one $A$ or one $B$ in $\Delta$ as a consequence of Sect. 7).

They are characterized by the indices $i, j, k$, boxes $\Delta^{\prime}, \Delta, \Delta^{\prime \prime}$ and points $z$ or $z_{1}$, $z_{2}$ in $\Delta$. We then choose:

$$
\begin{aligned}
r= & c n(\Delta)^{-1} M^{\jmath} M^{k} M^{-\varepsilon^{\prime}(i-j)} M^{-\varepsilon^{\prime}(i-k)} \\
& \times \exp \left\{(1-\eta) M^{-j} d\left(z_{1}, \Delta^{\prime}\right)+(1-\eta) M^{-k} d\left(z_{2}, \Delta^{\prime \prime}\right)\right\},
\end{aligned}
$$

with some small fixed $\varepsilon^{\prime}>0$ and $0<\eta<1$. The constant $c$ in front of the right-hand side will be chosen small enough as in Sect. 3. Points $z_{1}, z_{2}$ in $d\left(z_{1}, \Delta^{\prime}\right)$ or $d\left(z_{2} \Delta^{\prime \prime}\right)$ can be equally replaced by the box of $\mathbb{D}_{j}$, or $\mathbb{D}_{k}$ that contains $z_{1}$ or $z_{2}$.

For all vertices of this type, the analogue of the bound (19) holds equally as we now explain. To show this, a slight adaption of Lemma 1 of Sect. 3 will be used. Namely if $j, k$ denote supplementary discrete variables and if, on the other hand, $a$ is any strictly positive function (chosen equal to $M^{2 j}$ in the application), one has for any operator $H$ with regular kernel $H(x, j ; y, k)$,

\section{Lemma 3.}

where

$$
\begin{aligned}
\|H\| & <C(H)^{1 / 2} \\
C(H) & =\sup _{y, k} \sum_{j} \int d x E(x, j) a(y, k)|H(x, j ; y, k)|, \\
E(x, j) & =\sum_{k^{\prime}} \int|H(x, j ; y, k)| a^{-1}\left(y^{\prime}, k^{\prime}\right) d y^{\prime} .
\end{aligned}
$$


In the application $H=\sum_{i^{\prime}} H^{\left(i^{\prime}\right)}$, where each $H^{(i)}=H(x, j ; y, k)$ is of the form $A_{j}(x, z) B_{k}(z, y)$ and corresponds to a vertex produced at step $i$, so that $j, k \leq i+1$. Integrals over $y^{\prime}$ and then over $x$ are made in the same way as in Sect. 3. Putting in (53), summations over $k^{\prime}$ and $i^{\prime}$ are made, at any given $j$, with the help of the factors $M^{-\varepsilon^{\prime}\left(i^{\prime}-j\right)} M^{-\varepsilon^{\prime}\left(i^{\prime}-k^{\prime}\right)}: E$ is then shown to be bounded by cst $M^{-2 j}$. For any given $k$, the treatment of the remaining sum in (52) over $j$ and $i$ is made similarly. It is then shown that $C(H)$ is finite and less than any given constant if $c$ is small enough. b) For vertices in which $A_{j}$ or $B_{k}$, or both, are replaced by some first or second derivative, the choice of $r_{\alpha}$ is similar with the following changes: the factor $M^{j}$ is replaced by $M^{2 j}$ or $M^{3 j}$ respectively and $M^{k}$ is similarly replaced by $M^{2 k}$ or $M^{3 k}$. In view of the bounds (11), the previous argument on the norms of $\sum_{\alpha} r_{\alpha} V_{\alpha}$ is unchanged and these choice of $r_{\alpha}$ 's will later be adequate in power counting arguments.

c) We next consider vertices involving "badly localized" legs, of index $\geq i+1$ and start with vertices of the form $\partial^{2} A_{j}(., z) \partial^{2} B_{k}\left(z^{\prime},.\right) j, k \geq i+1, z, z^{\prime} \in \Delta\left(\in \mathbb{D}_{\imath}\right)$. They are first regrouped for each given $i$, and we are thus led to actual vertices of the form $\sum_{j>i} \partial^{2} A_{j} \sum_{k>i} \partial^{2} B_{k}$.

The radius $r_{\alpha}$, is then conveniently chosen of the form

$$
r_{\alpha}=c M^{6 i}(n(\Delta))^{-1} \text {. }
$$

If $H$ denotes here the sum of previous operators for given $i, \int \mid H(x, y \mid d y$ is bounded by

$$
\operatorname{cst} M^{6 i} \sum_{\Delta \in D_{\imath}} \frac{n(\Delta)}{n(\Delta)} \sum_{j, k} M^{-5 j} M^{-5 k} \int d y \exp \left\{-M^{-j} d\left(\Delta^{\prime}, \Delta\right)-M^{-k} d(\Delta, y)\right\}
$$

where $\Delta^{\prime}$ is the box that contains $x$. Using the fact that $\sum_{\Delta} e^{-M^{-\jmath} d\left(\Delta^{\prime}, \Delta\right)}$ is bounded by cst $M^{4(j-i)}$ (since boxes $\Delta$ belong to $D_{i}$ ) and that $\int e^{-M^{-k} d\left(\Delta^{\prime}, y\right)} d y$ is bounded by cst $M^{4 k}$ (for any given $\Delta$ ), one finally obtains factors $M^{-(k-i)} M^{-(j-i)}$ which allow one to make remaining summations over $k$ and $j(>i)$.

d) Remaining $V$ 's will be, after simple partial resummations, of the form

$$
L_{i, \Delta}=\left[\frac{1}{|\Delta|} \int_{\Delta} " \nabla A_{i+1}^{\prime \prime}(., z) d z\right]\left[\frac{1}{|\Delta|} \int_{\Delta} " \nabla B_{i+1}^{\prime \prime}(z, .) d z\right]
$$

where

$$
" \nabla A_{i+1}^{\prime \prime}=\nabla A_{i+1}+v_{i+1, \Delta} \nabla A_{i+2}+\cdots+\left(\prod_{m=i+1}^{k} v_{m, \Delta}\right) \nabla A_{k+1}+\cdots .
$$

It will also be convenient to rewrite it in the form:

$$
" \nabla A_{i+1}^{\prime \prime}(., z)=\sum_{\substack{j ; \\ m(\imath, z)-1 \leq j \leq \imath}}\left(1-v_{\jmath}(z)\right)^{\prime \prime} \nabla A_{j+1} "-\sum_{j ; m(i, z) \leq j \leq i} \nabla A_{j},
$$


where $m(i, z)$ is defined in (50). Let us now define $L_{i, \Delta}^{\prime}$ and $L_{i, \Delta}^{\prime \prime}$ as follows:

$$
\begin{aligned}
L_{i, \Delta}^{\prime}= & \frac{1}{|\Delta|} \int_{\Delta} \sum_{m(i, z)-1 \leq j \leq i}\left(1-v_{j}(z)\right)^{2 \prime \prime} \nabla A_{\jmath+1}^{\prime \prime}(., z) \\
& " \nabla B_{j+1}^{\prime \prime}(z, .) M^{-\varepsilon^{\prime}(i-m(i, z))} d z, \\
L_{i, \Delta}^{\prime \prime}= & \frac{1}{|\Delta|} \int_{\Delta} \sum_{m(i, z) \leq \jmath \leq i} \nabla A_{j}(., z) \nabla B_{\jmath}(z, .) M^{-\varepsilon^{\prime}(i-m(i, z))} d z,
\end{aligned}
$$

where $\varepsilon^{\prime}>0$ will later be chosen sufficiently small. $L_{i, \Delta}, L_{i, \Delta}^{\prime}$ and $L_{i, \Delta}^{\prime \prime}$ are all positive operators. We then state:

\section{Lemma 4.}

$$
L_{\imath, \Delta} \leq \operatorname{cst}\left(\varepsilon^{\prime}\right)\left[\frac{1}{|\Delta|} \int_{\Delta} M^{2 \varepsilon^{\prime}(i-m(i, z))} d z\right]\left(L_{i, \Delta}^{\prime}+L_{\imath, \Delta}^{\prime \prime}\right)
$$

Proof. We substitute (57) in (55) and then apply Schwarz inequality on each term of the sum obtained. The sum over one of the indices is made by using a factor $M^{-\varepsilon^{\prime}(i-m(z))}$. QED

We now explain how the relevant norm $\left\|\sum_{\alpha} \gamma_{\alpha} L_{\alpha}\right\|$ is bounded for a suitable choice of $r_{\alpha}$. Here there is one $\alpha$ for each choice of: an index $i$, a box $\Delta\left(\Delta \in D_{\imath}\right)$ and one of the at most $2 n(\Delta)$ operators of the form $L_{\imath, \Delta}$ that can be produced; $r_{\alpha}$ will be chosen, as is natural, to depend only on $i$ and $\Delta$. As a matter of fact, it is sufficient for our purposes to treat $\sum_{\alpha}\left(\operatorname{Re} \gamma_{\alpha}\right) L_{\alpha}$, which is bounded in the sense of operators by $\sum_{\alpha} r_{\alpha} L_{\alpha}$. In view of Lemma 2, the latter itself satisfies the bound,

$$
\begin{aligned}
\sum_{\alpha} r_{\alpha} L_{\alpha} \leq & \sum_{i, \Delta}\left[n(\Delta) r_{i, \Delta} \operatorname{cst}\left(\varepsilon^{\prime}\right)\left(\frac{1}{|\Delta|} \int_{-\Delta} M^{2 \varepsilon^{\prime}(\imath-m(i, z))} d z\right) M^{-4 i}\right] \\
& \times\left(M^{4 i} L_{i, \Delta+}^{\prime} M^{4 \imath} L_{i, \Delta}^{\prime \prime}\right) .
\end{aligned}
$$

We then choose $r_{i, \Delta}$ so that the first bracket in the right-hand side of (61) will be less than some small constant independent of $i, \Delta$, namely:

$$
r_{\imath, \Delta}=\operatorname{cst} c(n(\Delta))^{-1} M^{4 i}\left[\frac{1}{|\Delta|} \int_{\Delta} M^{2 \varepsilon^{\prime}(\imath-m(\imath, z))} d z\right]^{-1} .
$$

We then note that

(i)

$$
\begin{aligned}
\sum_{i, \Delta} M^{4 i} L_{\imath, \Delta}^{\prime} & \leq \sum_{\jmath} \int\left(1-v_{j}(z)\right)^{2 \prime \prime} \nabla A_{\jmath+1}^{\prime \prime}(., z)^{\prime \prime} \nabla B_{\jmath+1}^{\prime \prime}(z, .) d z \\
& \leq \sum_{\jmath} \int\left(1-v_{\jmath}(z)^{2}\right)^{\prime \prime} \nabla A_{j+1}^{\prime \prime}(., z)^{\prime \prime} \nabla B_{j+1}^{\prime \prime}(z, .) d z
\end{aligned}
$$


The operators in the right-hand side of (63) in $\sum_{j}$ are those occurring in the resolvent with a coefficient $\varepsilon_{i+1}$ that will remain close to the initial given $\varepsilon$, as a consequence of the initial introduction of the supplementary term $\varepsilon \nabla A . \nabla B$. This fact will allow one to "dominate" the corresponding contributions.

(ii) The contribution associated to the parts $L_{\imath, \Delta}^{\prime \prime}$ can be bounded in norm by

$$
\sum_{\substack{i, j \\ m(i, z) \leq j \leq i}} \int \nabla A_{j}(., z) \nabla B_{j}(z, .) M^{-\varepsilon^{\prime}(i-m(i, z))} d z .
$$

The sum over $i$ (at given $j$ ) is controlled by the factor $M^{-\varepsilon(i-m(i, z))}$. A bound on relevant norms is then obtained from Lemma 3.

\subsection{Bounds on F and Related Results}

As a result of previous analysis, the 2-point function $F$ appears, for any given $\varrho$, as a sum of contributions $F_{\beta}$ associated with diagrams $\beta$ (connected components with adequate specifications). Each $F_{\beta}$ is an integral, over variables $h, v, t, z$, of an integrand satisfying bounds including essentially the following factors:

$$
\begin{aligned}
& \prod_{\Delta}\left(\frac{1}{|\Delta|} \int_{\Delta} M^{2 \varepsilon^{\prime}(i-m(i, z))} d z\right]^{O(1) n(\Delta)} \prod_{\Delta}(n(\Delta) !)^{O(1)} \\
& \times \int \prod_{\Delta}\left|\frac{1}{|\Delta|} \int_{\Delta} \sigma(u) d u\right|^{s(\Delta)} d \mu(\vec{\sigma}) \\
& \prod_{\substack{4-\operatorname{leg} \\
\text { vertces } \not}} g_{m(\not{Z})} M^{0(1) \varepsilon^{\prime}\left(i\left(\mathscr{Z}^{\prime}\right)-m\left(\mathscr{Z}^{\prime}\right)\right)} \prod_{\substack{\text { well localzzed } \\
\text { legs }}} M^{-(j(\mathrm{leg}))(1+\text { number of derivatives })} \\
& \prod_{\substack{\text { badly localized } \\
\text { legs }}} M^{-\jmath_{\operatorname{leg}}(1+\text { number of derivatives })} \\
& \text { (products of factors } \left.\left(z-z^{\prime}\right)\right) \prod_{i} \prod_{\left(\Delta, \Delta^{\prime}\right) \in P_{\imath}} \exp \left\{-M^{-i}(1-2 \eta) d\left(\Delta, \Delta^{\prime}\right)\right\} \text {. }
\end{aligned}
$$

where $s(\Delta)$ is the number of mean values of $\sigma$ in $\Delta, s(\Delta) \leq 5+(3 / 2) n(\Delta)$ and $P_{i}$ is the set of the pairs of $\mathbb{D}_{i}$ with their $h$ 's different from zero. Products over $\Delta$ run over boxes $\Delta$ of the diagram under consideration. The second factor $(n(\Delta) !)^{0(1)}$ arises from the fact that each factor $1 / r$ gives one $n(\Delta)$ and that there might be (at most) $2 n(\Delta)$ such factors for each given $\Delta$.; for each vertex $\mathscr{V}, i(\mathscr{V})$ is the largest index $i$ such that there has been a derivative at that stage and $j(\mathrm{leg})$ is:

for a well localized leg equal to its index,

for a badly localized leg belonging to a vertex $\mathscr{V}$ equal to $i(\mathscr{V})$,

$m(\mathscr{Y})=m\left(i(\mathscr{Y}), z_{\mathscr{V}}\right)$ for a vertex $\mathscr{T}$ localized at $z_{\mathscr{V}}$. Finally, factors $\left(z-z^{\prime}\right)$ that have been indicated are all those arising from e.g. the renormalization procedure, involved in correction terms after replacement of some $z_{\beta}$ by $z_{\alpha}, \ldots$.

The following lemmas will be useful 


\section{Lemma 5.}

$$
\left[\prod_{i} \prod_{\Delta \in D_{\imath}}\left(\frac{1}{\Delta} \int_{\Delta} \sigma\right)^{2 s(\Delta)} d \mu(\sigma)\right]^{1 / 2} \leq \prod_{i} \prod_{\Delta}(\mathrm{cst})^{n(\Delta)}\left(M^{-2 i}\right)^{s(\Delta)}[(3 / 2) n(\Delta)] ! .
$$

Factors of the form shown in the left-hand side of (64) will arise from Schwarz inequalities. Lemma 5 is proved by explicit contractions, e.g.

$$
\begin{aligned}
\int \frac{1}{\Delta} \int_{\Delta} \sigma \frac{1}{\left|\Delta^{\prime}\right|} \int_{\Delta^{\prime}} \sigma d \mu(\sigma) & =\frac{1}{\Delta} \quad \text { if } \Delta^{\prime} \subset \Delta \\
& =0 \text { if } \Delta \cap \Delta^{\prime} \text { empty }
\end{aligned}
$$

where $\Delta \in \mathbb{D}_{\imath}, \Delta^{\prime} \in \mathbb{D}_{j}$, as a straightforward consequence of the $\delta$ functions in $\int \sigma_{\imath} \sigma_{j} d \mu(\vec{\sigma})$. In the general case, it is convenient to start by contracting $\sigma$ 's corresponding to the smallest boxes. QED

The $n(\Delta)$ !'s will be as before controlled by Lemma 2 .

We will extensively use the standard inequality (see [22]):

$$
\sum_{\beta}\left|\mathscr{A}_{\beta}\right| \leq \sup _{\beta}\left(a_{\beta}\left|\mathscr{A}_{\beta}\right|\right) \quad \text { if } \quad \sum_{\beta} a_{\beta}^{-1} \leq 1 \quad\left(a_{\beta}>0\right)
$$

We will apply (66) to the sum over all terms that contribute to $F$ (i.e. all connected components with adequate specifications) first for given positions of all boxes involved. Summation over the latter is made in turn later.

A convenient choice of $a_{\beta}$ is

$$
\begin{aligned}
a_{\beta} & =\prod_{\substack{\text { explicit } A \text { or } B^{\prime} \text { s } \\
\text { n each slice } \\
\text { joning boxes } \Delta, \Delta^{\prime}}} \exp \left\{\varepsilon^{\prime} M^{-i} d\left(\Delta, \Delta^{\prime}\right)\right\} \\
& \times \prod_{\substack{\Delta \in \text { connected } \\
\text { component } \beta}} 0(1) \prod_{\text {vertices }}\left(O(1) M^{\varepsilon^{\prime} h(\mathscr{\prime})}\right),
\end{aligned}
$$

where: $h(\mathscr{V})=i(\mathscr{V})-u(\mathscr{V})$, where $u(\mathscr{V})=($ the smallest index of the legs of $\mathscr{V})$.

The fact that $\Sigma a_{\beta}^{-1}$ is finite will be established in the usual ways. The exponential fall-off factors in distances $d\left(\Delta, \Delta^{\prime}\right)$ allow one to control the number of terms generated by the cluster expansion. For $i(\mathscr{V})$ given, the factors $M^{-\varepsilon h(\mathscr{P})}$ allow one to control the sum over the slices of previous derivatives (a vertex $\mathscr{V}$ could have been derived at any slice between $i(\mathscr{V})$ and $i(\mathscr{V})-h(\mathscr{V})$ ). We note that, though we have mainly considered the 2 point function $F$ for definiteness, the analysis is similar for $N$-point functions and the discussion below is given for general $N$.

Let us come back to the $N$ external legs $A$ and $B$ which appear in the expression of the (connected) $N$-point function (9). Each $A$ or $B$ has been decomposed into $\sum_{i} A^{i}$ or $\sum_{\imath} B^{\imath}$. We first treat separately contributions corresponding to given values $i_{1}, \ldots, i_{N}$. (We shall later use factors $M^{i_{1}}, \ldots, M^{i_{N}}$ occurring in bounds for each $i_{k}$.) We then note that by construction:

$$
\operatorname{Inf}_{\text {vertices }} u(\mathscr{V}) \leq i_{1}, \ldots, i_{N} \leq \operatorname{Sup}_{\text {vertices }} u(\mathscr{T}) .
$$


In fact, if e.g. $\operatorname{Inf} u(\mathscr{V})$ is equal to some index $\gamma$, all variables $v_{\Delta}$ of index $\gamma-1$ vanish so that corresponding contributions also vanish. Formula (68) allows one to show that $\sum_{\beta} a_{\beta}^{-1}$ is indeed finite for given $i_{k}$ 's.

From (66), a bound on $\sum_{\beta}\left|\mathscr{A}_{\beta}\right|$ is obtained by taking the Sup over $\beta$ of the following bounds on $\left|a_{\beta} \mathscr{b}_{\beta}\right|$ :

$$
\begin{aligned}
& \int \prod_{\text {vertices }} d z\left(M^{-j}\right)^{N_{1}(j)}\left(M^{-i}\right)^{N_{2}(i)} \prod_{\text {vertices }} \operatorname{cst} \sqrt{g}_{m(\mathscr{V})} M^{0(1) \varepsilon^{\prime} h(\mathscr{V})} \\
& \prod_{\Delta}\left(\frac{1}{\Delta} \int_{\Delta} M^{\varepsilon(i-m(i, z))} d z\right)^{0(1) n(\Delta)} \\
& \times \prod_{\substack{\text { explict } A \text { and } B^{\prime} s \\
\text { of the cluster expansion }}} e^{-\operatorname{cst} M^{-\imath} d\left(\Delta, \Delta^{\prime}\right)} \prod \text { factors }\left(z-z^{\prime}\right)
\end{aligned}
$$

when $N_{1}(j)$ is the number of $A, B$ and derivatives of index $j$ corresponding to "well localized" legs ( $j \leq i+1$ if $i$ is the last stage where some procedure has been applied to one of the legs of the vertex), and $N_{2}(i)$ is the number of $A, B, \nabla$ corresponding to $A$ or $B$ of slice $>i$ that have been eliminated, or to $A, B$ in terms " $\nabla A_{i+1}^{\prime \prime} " \nabla B_{i+1}^{\prime \prime}$ that have been "dominated," or in terms $\sum_{k \geq i} \nabla^{2} A_{k}, \sum_{k \geq \imath} \nabla^{2} B_{k}$.

Remark

$$
\frac{g_{m(\imath, z)}}{g_{i}}<\operatorname{cst} \sqrt{\frac{i}{m(i, z)}}<\operatorname{cst}\left(\varepsilon^{\prime \prime}\right) M^{\varepsilon^{\prime \prime}(i-m(i, z))}
$$

where the inequality on the left is due to results on the behaviour of $g_{i}$. The rough inequality on the right, valid for any $\varepsilon^{\prime \prime}>0$, will be sufficient for our purposes.

We next use

\section{Lemma 6.}

$$
\prod_{i} \prod_{\Delta \in \mathbb{D}_{\imath}}\left(\frac{1}{|\Delta|} \int_{\Delta} M^{\varepsilon(\imath-m(i, z))} d z\right)^{4 n(\Delta)} \leq \prod_{i} \prod_{\Delta \in \mathbb{D}_{i}}\left[0(1)(n(\Delta) !)^{4}\right] .
$$

Remark. The factor $n(\Delta) !^{4}$ will later be "absorbed" by the exponential fall-off factors, following Lemma 2.

Proof of Lemma 6

$$
\begin{aligned}
& \prod_{i} \prod_{\Delta \in \mathbb{D}_{i}}(n(\Delta) !)^{-4}\left(\int \frac{1}{|\Delta|} \int_{\Delta} M^{\varepsilon(i-m(i-z))} d z\right)^{4 n(\Delta)} \\
& \quad \leq \exp \left[\sum_{i} \sum_{\Delta \in \mathbb{D}_{i}} \frac{1}{|\Delta|} \int_{\Delta} M^{\varepsilon(i-m(\imath, z))} d z\right]
\end{aligned}
$$


On the other hand, denoting below $\Lambda_{i}$ the set of boxes of $P_{i}$,

$$
\begin{aligned}
& \sum_{i} \sum_{\Delta \in \mathbb{D}_{i}} \frac{1}{|\Delta|} \int_{\Delta} M^{\varepsilon(i-m(i, z))} d z \leq \sum_{i} \sum_{\Delta \in \mathbb{D}_{\imath}} \int_{\Delta} M^{-(4-\varepsilon) i-\varepsilon m(i, z)} d z \\
& =\sum_{\imath} \int_{\Lambda_{\imath}} M^{-(4-\varepsilon) i-\varepsilon m(i, z)} d z \leq \sum_{i} \sum_{j \leq i} \int_{\Lambda_{i} \cap \Lambda_{\jmath}} M^{-(4-\varepsilon)(i-j)} M^{-4 j} d z \\
& \leq \sum_{j} \sum_{\Delta \subset \Lambda_{j}} \sum_{\imath \geq j} M^{-(4-\varepsilon)(\imath-j)} \leq \sum_{\Delta} O(1)
\end{aligned}
$$

We now briefly describe the following usual procedures that can be used to get bounds on the $N$ point function $F_{\imath_{1},,_{N},}$, with external $A$ and $B$ 's of indices $i_{1}, \ldots, i_{N}$. The following inequalities will be useful:

$$
\exp \left\{-M^{-i}(x-y)\right\} \leq \prod_{i=j}^{\varrho} \exp \left\{-0(1) M^{-j|x-y|}\right\}
$$

Equation (70) will allow one, given an exponential fall-off factor at a given slice $i$, to attribute similar factors to all slices $j \geq i$. These exponential decrease factors allow one to get rid of factors $z-z^{\prime}$ modulo factors $M^{j}$ :

$$
\left|z-z^{\prime}\right| e^{-0(1) M^{-j}\left|z-z^{\prime}\right|}<\operatorname{cst} M^{j}
$$

where $j$ is the slice at which the operation giving rise to $z-z^{\prime}$ has been made: integration over variables $z$ is then made inductively from slice 1 to $\varrho$ with, at each stage $i$, a "fixed" vertex for each connected component of slices less than $i$. Integration over other vertices is made with the help of exponential fall-off factors which allow to sum over the box containing each vertex. There is no fixed vertex if the component contains some external legs. Factors $M^{4 i}$, namely the volume of the box in which a point has been integrated (in slice $i$ ) are obtained. They are conveniently written in the form:

$$
M^{4 i}=M^{4} \varrho \prod_{j=i+1}^{\varrho} M^{-4}
$$

Factors $M^{i}$ or $M^{-i}$, arising e.g. from (71) or from gradients respectively, are similarly written

$$
M^{j}=M^{\varrho} \prod_{j^{\prime}=j+1}^{\varrho} M^{-1}, \quad M^{-i}=M^{-\varrho} \prod_{j=i+1}^{\varrho} M
$$

Factors $M^{\varrho}$ and $M^{-\varrho}$ cancel each other and one finally obtains the following powers of $M$ :

$$
\begin{aligned}
& M^{-i_{1}} \ldots M^{-i_{N}} \prod_{j} \prod_{\begin{array}{c}
\text { connected } \\
\text { components } G_{j}
\end{array}}\left(M^{-4\left(\left[\text { number of vertices of } G_{j}\right]-1\right)}\right. \\
& \left.\times M^{-\left[\text {number of factors }\left(z-z^{\prime}\right)\right]} M^{[\text {number }(A, B, \text { gradients })]}\right)
\end{aligned}
$$


which can itself be bounded by

$$
M^{-i_{i}} \ldots M^{-i_{N}} \prod_{j} \prod_{\substack{G_{j} \text { that do not include } \\ \text { external legs }}} M^{-\left(4-e\left(G_{j}\right)\right)} \prod_{\substack{G_{j} \text { containing } \\ \text { external legs }}} M^{-e\left(G_{j}\right)}
$$

where $e\left(G_{j}\right)$ is the number of outgoing legs (legs of indices larger than $j$ ) of $G_{j}$, and in turn by

$$
M^{-i_{1}} \ldots M^{-\imath_{N}} \prod_{\text {vertices } \mathscr{V}} M^{-h(\mathscr{V}) / 5}
$$

The final uniform bound on $F_{i_{1}, \quad,{ }_{N}}$ (independent of $\varrho$ ) follows:

$$
\begin{aligned}
& \left|F_{i_{1}, \quad, i_{N}}\left(x_{1}, \ldots, x_{N}\right)\right|<\operatorname{cst} M^{-i_{1}} \ldots M^{-i_{N}} \\
& \quad \times \sup _{\beta} \prod_{\text {vertices } \mathscr{V}}\left[\operatorname{cst} M^{\left(-1 / 5+O(1) \varepsilon^{\prime}\right) h(\mathscr{V})} \sqrt{g_{i(\mathscr{V})}}\right] \\
& \quad \times \exp \left\{-\operatorname{cst} M^{-\sup \left(i_{1}, \quad,_{N}\right)} L\left(x_{1}, \ldots, x_{N}\right)\right\}
\end{aligned}
$$

where $L$ is the minimal length of all connected trees joining $x_{1} \ldots x_{N}$ and possibly intermediate points.

As already mentioned, convergence in the $p \rightarrow \infty$ limit is established similarly. At that stage the following bounds are obtained (in the $\varrho \rightarrow \infty$ limit) on e.g. the 2 point function

(i) $i=i_{2}=i \quad \operatorname{cst} M^{-2 i} e^{-\operatorname{cst} M^{-\imath}|x-y|}$.

(ii) $i_{1} \neq i_{2}$. There are in this case at least 2 couplings and one obtains the bound

$$
\begin{aligned}
\left|F_{i_{1}, i_{2}}(x, y)\right|< & \operatorname{cst} M^{-i_{1}} M^{-i_{2}} M^{-\left((1 / 5)-O(1) \varepsilon^{\prime}\right)\left|i_{1}-i_{2}\right|} g_{\inf \left(i_{1}, i_{2}\right)} \\
& \times \exp \left\{-\operatorname{cst} M^{-\sup \left(i_{1}, i_{2}\right)}|x-y|\right\} .
\end{aligned}
$$

Similarly, the following bound is obtained for the 4-point connected function

$$
\begin{aligned}
& \left|F_{i_{1}, \quad,,_{4}}\left(x_{1}, \ldots, x_{4}\right)\right| \\
& \quad<\operatorname{cst} M^{-2 i_{1}} \ldots M^{-2 i_{4}} g_{\inf \left(i_{1}, \quad, i_{4}\right)} M^{\left(-(1 / 5)+O(1) \varepsilon^{\prime}\right)}\left|\sup \left(i_{1}, \ldots, i_{4}\right)-\inf \left(i_{1}, \ldots, i_{4}\right)\right| \\
& \quad \times \exp \left\{-\operatorname{cst} M^{-\sup \left(i_{1}, \quad,,_{4}\right)} L\left(x_{1}, \ldots, x_{4}\right)\right\} .
\end{aligned}
$$

However, these results are not sufficient for the analysis of the large distance behaviour of $F$ to any order. To get better results (at order $n$ in $(\ln |x-y|)^{-1}$ ) it is sufficient to put aside, in the expansion, terms with a number $n$ of vertices less than any given $n_{0}\left(n \leq n_{0}\right)$. The remainder will be bounded as above. The bound will now include the factor:

$$
\left.g_{\text {inf }\left(i_{1},\right.}^{n_{0} / 2}, i_{N}\right) .
$$

Bounds on the terms that have been put aside include factors $\left(g_{\text {inf }\left(i_{1}, i_{N}\right)}\right)^{n}, n \leq n_{0}$. The leading behaviour is then obtained up to order $n_{0} / 2$. 


\subsection{Large Distance Behaviour: Proof of the Theorem.}

The large distance behaviour will then be obtained up to order $n_{0} / 2$. For the 2-point function, the theorem will be obtained, after resummation over $i_{1}, i_{2}$. With so far $i_{1}$, $i_{2}$ given, one has:

(i) $i_{1}=i_{2}=i$. The lowest order contribution $\bullet \overline{A_{i} B_{i}} \bullet$ gives a result in $M^{-2 \imath} e^{-M^{-\imath}|x-y|}$. The bound on the remainder includes the further factor $g_{i}^{2}$ of the order of $1 / i$.

(ii) $i_{1} \neq i_{2}$. These contributions will be included in higher order terms when summation over $i_{1}, i_{2}$ is made as now explained.

Let us in fact consider the actual 2-point function $F(x, y)=\sum_{i_{1}, i_{2}} F_{i_{1}, i_{2}}(x, y)$.

Explicit lower order terms are resummed, while bounds obtained previously allow one to treat remaining sums. Given $x, y$, let $i=i(x, y)$ be the best integer such that $|x-y| \sim M^{i}$. Let $j=\operatorname{Sup}\left(i_{1}, i_{2}\right)$. For $j \leq i$, the exponential fall-off factor $\exp \left\{-M^{-j}|x-y|\right\}$ is used and provides a very small factor $\mathrm{e}^{-M^{i-\jmath}}$, allowing one to make the summation with a result much smaller than explicit terms.

For $j>i$ and if e.g. $i_{1}, i_{2}>i$, one may write: $M^{-i_{1}} M^{-i_{2}}=$ $M^{-2 i} M^{-\left(i_{1}-i\right)} M^{-\left(i_{2}-i\right)}$, where $M^{-2 \imath} \cong 1 /|x-y|^{2}$ and factors $M^{-\left(i_{1}-i\right)}$ and $M^{-\left(\imath_{2}-i\right)}$ allow one to sum over $i_{1}, i_{2}$. A similar analysis holds in other cases.

The result stated in Theorem 2 is then obtained by estimating the leading contributions to $F(x-y)$ :

$$
\begin{aligned}
F(x, y) & =\frac{1+O\left(g_{\imath}^{4}\right)}{\zeta_{i}|x-y|^{2}}=\frac{1}{\zeta_{\text {ren }}|x-y|^{2}}\left[\frac{1+O\left(g_{i}^{4}\right)}{1+\frac{\zeta_{\imath}-\zeta_{\text {ren }}}{\zeta_{\text {ren }}}}\right] \\
& =\frac{1}{\zeta_{\text {ren }}|x-y|^{2}}\left[1+\frac{\sum_{j>i} \delta \zeta_{j}}{\zeta_{\text {ren }}}+O\left(g_{\imath}^{4}\right)\right] .
\end{aligned}
$$

We use now that $\delta \zeta_{j}=a g_{j}^{4} \ln M+$ higher terms, where $a$ is the constant corresponding to the lowest order diagram contributing to the wave function renormalization, and get:

$$
F(x, y)=\frac{1}{\zeta_{\text {ren }}|x-y|^{2}}\left(1+a \ln (M) \sum_{j>\imath}\left(\frac{1}{-\beta_{2} j \ln M+\frac{\beta_{3}}{\beta_{2}} \ln j+C^{\prime}}\right)^{2}+O\left(i^{-2}\right)\right) .
$$

By summing over $j$, we obtain with $i \approx \ln |x-y| / \ln M$ :

$$
F(x, y)=\frac{1}{\zeta_{\text {ren }}|x-y|^{2}}\left(1+\frac{\lambda_{1}}{i \ln M}+\frac{\lambda_{2} \ln i}{(i \ln M)^{2}}+O\left(i^{-2}\right) O\left(1 / g^{2}\right)\right),
$$

where $\lambda_{1}$ and $\lambda_{2}$ are depending only on $a, \beta_{2}$ and $\beta_{3}$.

Remark More generally, for an $N$-point function the natural expansion that one obtains is an expansion in terms of the effective parameters and where subdiagrams are renormalized (by definition here a subdiagram has its internal legs of higher momentum than the external ones) $[18,15]$. The large distance behaviour at a given order is then obtained by resumming the first order contributions and using the behaviour of these effective parameters, following the analysis of [19]. 
Acknowledgements We are pleased to thank B. Duplantier for discussions at the origin of this work and for useful remarks We also thank the referee for a careful reading of the manuscript and a large number of useful remarks.

\section{References}

1. Edwards, S.F.: Proc. Phys. Soc. 85, 613 (1965)

2. Edwards, S.F.: Proc. Phys Soc. 88, 265 (1966)

3. de Gennes, P.G.: Phys. Lett. 38A, 339 (1972)

4. Duplantier, B.: C.R Acad. Sci. Paris 290 B, 199 (1980)

5. Brézin, E, Le Guillou, J C., Zinn-Justin, J : Phase transitions and critical phenomena. Domb, C, Green, M.S., (eds), vol. 6, New York: Academic Press, 1976, p 127; Zinn-Justin, J.: Quantum field theory and critical phenomena. Oxford: Oxford Univ. Press, 1989, ch. 24 and references therein to original works.

6 Duplantier, B : Nucl Phys. 275 B, 319 (1986): Commun Math Phys. 117, 279 (1988)

7 Varadhan, S R.S.: Appendix in Symanzik, K, Local quantum theory, Varenna 1968, New York: Academic Press, 1970, p. 285

8 Westwater, M.J.: Commun. Math. Phys. 72, 131 (1980)

9. Lawler, G.F.: Commun. Math. Phys. 86, 539 (1982)

10. Aizenman, M.: Commun. Math. Phys. 97, 91 (1985)

11. Feldman, J., Magnen, J., Rivasseau, V., Sénéor, R.: Commun Math Phys. 109, 437 (1987)

12. Gawedzki, K., Kupiainen, A : Commun. Math. Phys 99, 197 (1985)

13 Duplantier, B.: Private communication

14. Glimm, J., Jaffe, A.: Quantum physics Berlin, Heidelberg, New York: Springer 1981, 1987, and references therein to original works.

15. Rivasseau, V : From perturbative to constructive renormalization. Princeton: Princeton Univ Press, 1990

16 Duneau, M., Iagolnitzer, D., Souillard, B : Commun. Math. Phys. 31, 191 (1973)

17. Iagolnitzer, D., Magnen, J : Commun Math Phys 110, 51 (1987)

18. Feldman, J, Magnen, J., Rivasseau, V., Sénéor, R.: Commun Math Phys. 103, 67 (1986)

19 Iagolnitzer, D., Magnen, J.: Commun. Math. Phys. 119, 609 (1988)

20. Brydges, D., Spencer, T : Commun. Math. Phys. 97, 125 (1985)

21. Hara, T., Slade, G.: Commun. Math. Phys. 147, 101 (1992)

22. Glimm, J., Jaffe, A.: Fortschr. Phys. 21, 327 (1973)

Communicated by M. Aizenman 
